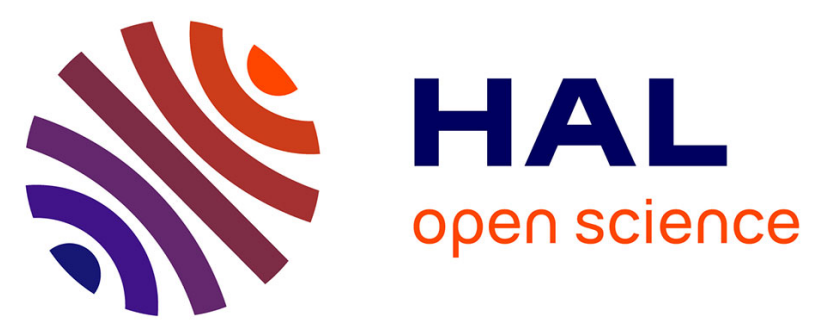

\title{
Bottom-up and top-down control of dispersal across major organismal groups
}

Emanuel A Fronhofer, Delphine Legrand, Florian Altermatt, Armelle Ansart, Simon Blanchet, Dries Bonte, Alexis Chaine, Maxime Dahirel, Frederik de Laender, Jonathan de Raedt, et al.

\section{To cite this version:}

Emanuel A Fronhofer, Delphine Legrand, Florian Altermatt, Armelle Ansart, Simon Blanchet, et al.. Bottom-up and top-down control of dispersal across major organismal groups. Nature Ecology \& Evolution, 2018, 2 (12), pp.1859-1863. 10.1038/s41559-018-0686-0 . hal-01936819

\section{HAL Id: hal-01936819 https://hal-univ-rennes1.archives-ouvertes.fr/hal-01936819}

Submitted on 4 Dec 2018

HAL is a multi-disciplinary open access archive for the deposit and dissemination of scientific research documents, whether they are published or not. The documents may come from teaching and research institutions in France or abroad, or from public or private research centers.
L'archive ouverte pluridisciplinaire HAL, est destinée au dépôt et à la diffusion de documents scientifiques de niveau recherche, publiés ou non, émanant des établissements d'enseignement et de recherche français ou étrangers, des laboratoires publics ou privés. 


\section{Bottom-up and top-down control of dispersal across major organismal groups}

Emanuel A. Fronhofer ${ }^{1,2,3 *}$, Delphine Legrand ${ }^{5}$, Florian Altermatt ${ }^{1,2}$, Armelle Ansart ${ }^{4}$,

Simon Blanchet ${ }^{5,6}$, Dries Bonte ${ }^{7}$, Alexis Chaine ${ }^{5,8}$, Maxime Dahirel ${ }^{4,7}$, Frederik De Laender $^{9}$, Jonathan De Raedt ${ }^{9,10}$, Lucie di Gesu ${ }^{6}$, Staffan Jacob ${ }^{11}$, Oliver Kaltz ${ }^{3}$, Estelle

Laurent $^{11}$, Chelsea J. Little ${ }^{1,2}$, Luc Madec ${ }^{4}$, Florent Manzi ${ }^{3}$, Stefano Masier ${ }^{7}$, Felix

Pellerin ${ }^{6}$, Frank Pennekamp ${ }^{2}$, Nicolas Schtickzelle ${ }^{11}$, Lieven Therry ${ }^{5}$, Alexandre Vong ${ }^{5}$, Laurane Winandy ${ }^{6}$ and Julien Cote ${ }^{6}$

1 Eawag: Swiss Federal Institute of Aquatic Science and Technology, Department of Aquatic Ecology, Überlandstrasse 133, CH-8600 Dübendorf, Switzerland

2 Department of Evolutionary Biology and Environmental Studies, University of Zurich, Winterthurerstrasse 190, CH-8057 Zürich, Switzerland

3 ISEM, Université de Montpellier, CNRS, IRD, EPHE, Montpellier, France

4 Université Rennes 1, Centre National de la Recherche Scientifique (CNRS), UMR6553 EcoBio, F35042 Rennes cedex, France

5 Centre National de la Recherche Scientifique (CNRS), Université Paul Sabatier, UMR5321 Station d'Ecologie Théorique et Expérimentale (UMR5321), 2 route du CNRS, F-09200 Moulis, France.

6 Centre National de la Recherche Scientifique (CNRS), Université Paul Sabatier; UMR5174 EDB (Laboratoire Évolution \& Diversité Biologique), 118 route de Narbonne, F-31062 Toulouse cedex, France.

7 Ghent University, Dept. Biology, K.L. Ledeganckstraat 35, B-9000 Ghent, Belgium

8 Institute for Advanced Studies in Toulouse, Toulouse School of Economics, 21 allée de Brienne, 31015 Toulouse, France

9 University of Namur, Research Unit in Environmental and Evolutionary Biology, Rue de Bruxelles, 61, 5000 Namur, Belgium

10 Ghent University, Laboratory of Environmental Toxicology and Applied Ecology, Coupure Links 653, 9000 Ghent, Belgium

11 Univ. Catholique de Louvain, Earth and Life Inst., Biodiversity Research Centre, Croix du Sud 4 L7.07.04, Louvain-la-Neuve, Belgium

* Corresponding author. Orcid ID: 0000-0002-2219-784X

Keywords: context-dependent dispersal, predator-induced dispersal, predation risk, resource limitation, food chain, distributed experiment, metaexperiment

This is a post-peer-review, pre-copyedit version of an article published in Nature Ecology and Evolution. The final authenticated version is available online at:

http://dx.doi.org/10.1038/s41559-018-0686-0

Correspondence Details

Emanuel A. Fronhofer

Institut des Sciences de l'Evolution de Montpellier, UMR5554

Université de Montpellier, CC065, Place E. Bataillon, 34095 Montpellier Cedex 5, France

phone: +33 (0) 467143182

email: emanuel.fronhofer@umontpellier.fr 


\begin{abstract}
Ecology and evolution unfold in spatially structured communities, where dispersal links dynamics across scales. Because dispersal is multi-causal, identifying general drivers remains challenging. In a coordinated distributed experiment spanning organisms from protozoa to vertebrates, we tested whether two fundamental determinants of local dynamics, top-down and bottom-up control, generally explain active dispersal. We show that both factors consistently increased emigration rates and use metacommunity modelling to highlight consequences on local and regional dynamics.
\end{abstract}


Dispersal is a life-history trait ${ }^{1}$ that fundamentally impacts spatial population and community ecology. ${ }^{2,3}$ By linking dynamics between local and regional scales via gene flow, dispersal also strongly determines evolutionary change. ${ }^{4}$ Dispersal is especially relevant in the context of current global changes $^{5}$ : increasingly fragmented landscapes, as well as shifting climatic conditions, may force organisms to disperse in order to survive and to maintain metacommunity and foodweb properties. ${ }^{6}$ However, dispersal is often grossly oversimplified in models ${ }^{5}$ a representation which is at odds with the growing awareness that dispersal must be considered in sufficient detail for a better understanding of ecology and evolution as well as for improving biodiversity forecasts. ${ }^{5,7}$

Understanding the causes and consequences of dispersal is challenging, because dispersal is a highly plastic trait that depends on multiple factors at both the intra- and interspecific level, ${ }^{8-10}$ such as resource availability, ${ }^{11,12}$ intraspecific densities ${ }^{13,14}$ or interspecific interactions, ${ }^{15,16}$ as illustrated by empirical work. Theoretical work has shown that context-dependent dispersal has important consequences in the context of intraspecific competition, ${ }^{17,18}$ predator-prey interactions ${ }^{19,20}$ and species coexistence, ${ }^{21}$ to name but a few examples.

The challenge is to uncover fundamental proximate drivers of dispersal, which are relevant to population and community dynamics, while simultaneously maintaining generality and tractability. We argue that dispersal is best understood and investigated within the relevant community setting where it is likely a function of the fundamental ecological forces that determine local population dynamics, including bottom-up (resource availability) and top-down (predation risk) impacts that regulate the focal species demography.

To investigate this hypothesis as well as to provide a general test of the ubiquity of context-dependent dispersal (CDD), we need synthetic datasets covering multiple species. Such datasets should be obtained using comparable methodology and, most importantly, should include responses to multiple drivers of dispersal simultaneously as these may interact, which can lead to non-additive effects. ${ }^{9,22}$ Such datasets have hitherto been largely lacking for dispersal. ${ }^{5,7}$ Therefore, we conducted a coordinated distributed experiment ${ }^{23,24}$ involving 7 laboratories across Europe and 21 species ranging from protozoa to vertebrates to test for bottom-up and top-down effects on dispersal, more specifically on the emigration phase of dispersal, ${ }^{25}$ in experimental two-patch systems. By designing the two-patch systems with connections between them to be 'hostile matrices', incompatible with sustained population survival, we test emigration decisions rather than routine movement (see Supplementary Information for details). The emigration phase is crucial, as it initiates dispersal, is readily controllable by behavioural decisions and therefore strongly determines the course of subsequent dispersal phases. ${ }^{8}$

We found that resource availability and predation risk, that is, the perceived presence of a predator based on chemical, visual and/or auditory cues, impacted emigration decisions across all study species 
(Fig. 1; Supplementary Table 2). The most parsimonious statistical model suggests that the effects of resource availability and predation risk were additive (Supplementary Table 2). While resource limitation led to a clear increase in emigration across all focal species (on average from approx. 9\% to 16\% without predation; relative importance of resource availability, i.e., sum of AICc weights of models in which the parameter occurs: 1.00), the effect of predation risk was overall weaker (on average from approx. $9 \%$ to $12 \%$ without resource limitation; relative importance of predation risk: 0.88$)$. The interaction between predation risk and resource availability suggested by the second ranked model $(\Delta \mathrm{AICc}=2.07 ; \mathrm{AICc}$ weight $=0.23$; see Supplementary Table 2) appeared to be only of marginal importance, as illustrated by the high overlap of distributions in Fig. 1.

In accordance with our results (Fig. 1b), we generally expected resource limitation to increase emigration rates in order to escape from low fitness environments. ${ }^{9}$ A post-hoc exploration of emigration responses for each species, estimated using log odds ratios (Supplementary Figure 2a, Supplementary Tables 3-4), confirmed this finding overall (the best model only includes the intercept; AICc weight = $0.55)$, while tentatively suggesting that the focal species' feeding strategy ${ }^{26}$ might have modulated this response (relative parameter importance: 0.23 ; second ranked model with $\Delta \mathrm{AICc}=2 ;$ AICc weight $=$ 0.20). While sit-and-wait and active capture foragers tended to respond less, grazers clearly responded more to resource limitation by increased emigration. We hypothesize that, if grazers rely on resources of limited mobility, local resource limitation reliably indicates low fitness expectations which should induce emigration. For both other foraging strategies, resources may be too mobile to reliably indicate (future) fitness expectations. However, we warn readers not to draw firm conclusions on this specific point. The strength of the effect is relatively weak and species are not evenly distributed across feeding strategies. By contrast, in the literature, little consensus exists on possible responses to predation risk, which has been suggested to depend on space use behaviour of predators and prey. ${ }^{19}$ Again, using a post-hoc exploration of emigration responses to predation, the intercept model ranked first (AICc weight $=0.15$, Supplementary Tables $5-6)$. However, as suggested by the second ranked model $(\Delta \mathrm{AICc}=0.15$; AICc weight $=0.14$ ) and the averaged model predictions (Supplementary Figure 2b), the direction of the effect of predation indeed depended somewhat on the relative space use of the focal species, that is, the extent of space routinely used by the focal species (e.g., a home range) relative to the predator's space use (Supplementary Figure 2b, Supplementary Table 5; relative importance of space use: 0.26 ) and the mode of dispersal of the focal species (terrestrial, aquatic or aerial dispersal; which imply characteristically different dispersal costs $;^{27}$ relative importance of dispersal mode: 0.33$)$. Finally, whether predators were generalists or specialists may also have impacted emigration responses (relative importance: 0.38), with specialist predators tentatively leading to higher emigration rates. However, these effects have to be interpreted cautiously, as the analysis is post-hoc and the first ranking model consistently included only 


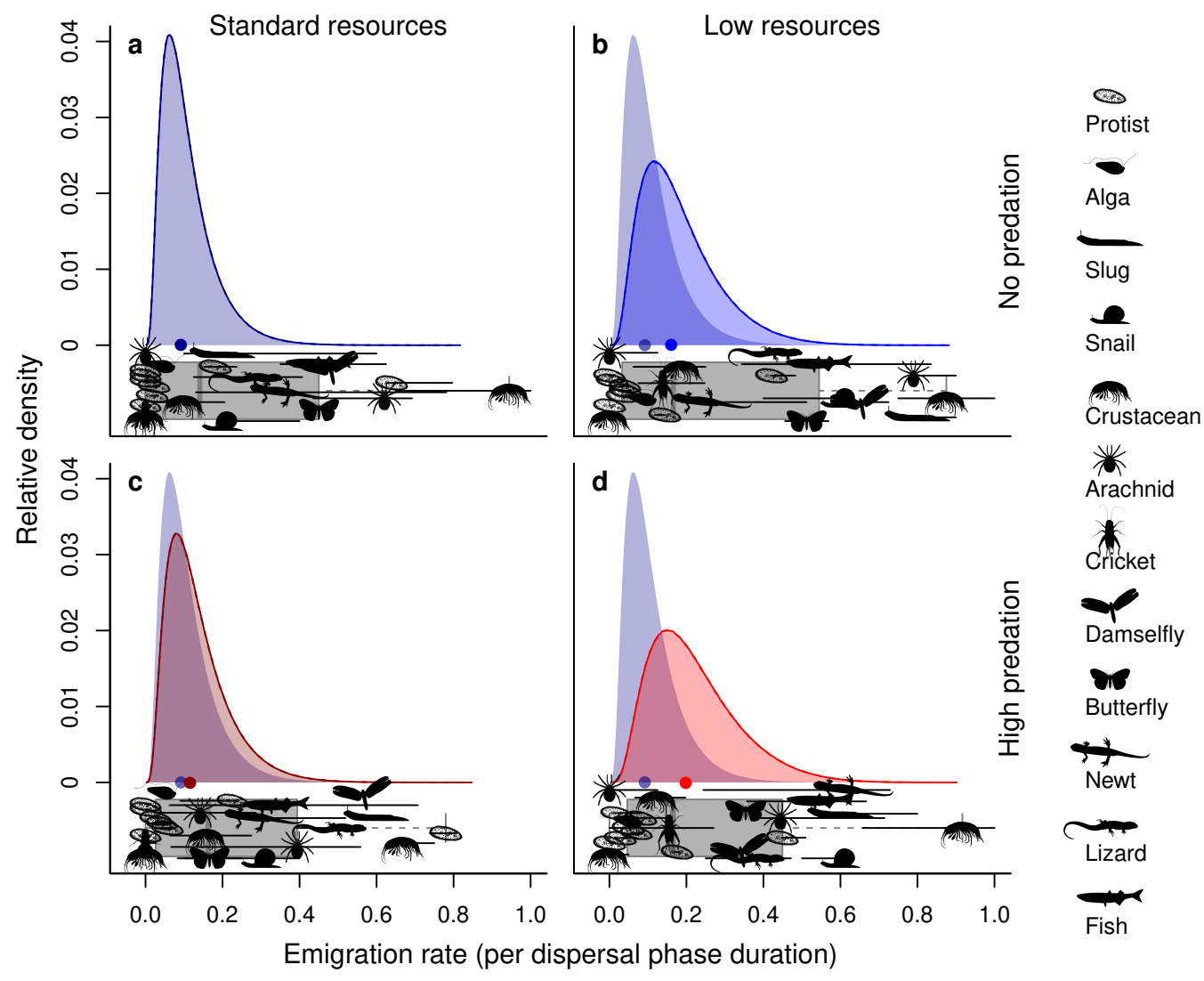

Figure 1: Effect of bottom-up resource limitation and top-down predation risk on emigration across 21 species, ranging from protists to vertebrates. AICc-based model selection on binomial GLMMs suggest an additive effect of predation risk and resource limitation (see Supplementary Table 2; Intercept (RA low, PRED no): $-1.65 \pm 0.69$; RA std.: $-0.64 \pm 0.11$; PRED yes $0.26 \pm 0.11)$. We show posterior predictive distributions (continuous lines and coloured shaded areas; dots represent medians of the distributions) of the most parsimonious, that is additive, model (lighter shades indicate resource limitation; blue: without predator cues; red: with predator cues). For pairwise differences between the posterior distributions see Supplementary Figure 1. For comparison all panels include the distribution of the reference scenario (a; standard resources and no predation; dark blue) and we additionally plotted the posterior predictive distributions of the model including the interaction between resource limitation and predation risk (dashed lines) which completely overlaps with the prediction of the additive model. Below the model predictions, we show observed median emigration rates (black animal symbol) and quartiles (corresponding black error line) per study species, as well as box plots across all species (grey; showing median, and quartiles, the whiskers extend beyond the quartiles by 1.5 times the interquartile range).

the intercept.

Shifting our focus from causes of dispersal to its consequences, we illustrate the potential impact of CDD in metacommunities using a simple food chain model that includes a basal resource, a focal consumer and a top-predator in analogy to the experiment (Fig. 2; for a sensitivity analysis see Supplementary Table 13 - 14 and Supplementary Figures $3-5$ ). Simultaneous resource- and predator-dependent emigration as found experimentally greatly reduced local fluctuations of population dynamics through time. At a regional metacommunity level, CDD dramatically reduced covariance between patch dynamics. Both of these effects are directly relevant to local and regional metacommunity stability ${ }^{28}$ as stability increases 
with smaller intrinsic fluctuations and less synchronous patch dynamics. Interestingly, CDD in the focal species did not only affect its own dynamics, but had cascading effects on the other trophic levels which highlights the importance of dispersal for driving species network dynamics. ${ }^{6}$ These results suggest that CDD could, via its stabilizing effect, reduce stochastic extinction risk in metacommunities, at least for lower and intermediate trophic levels.

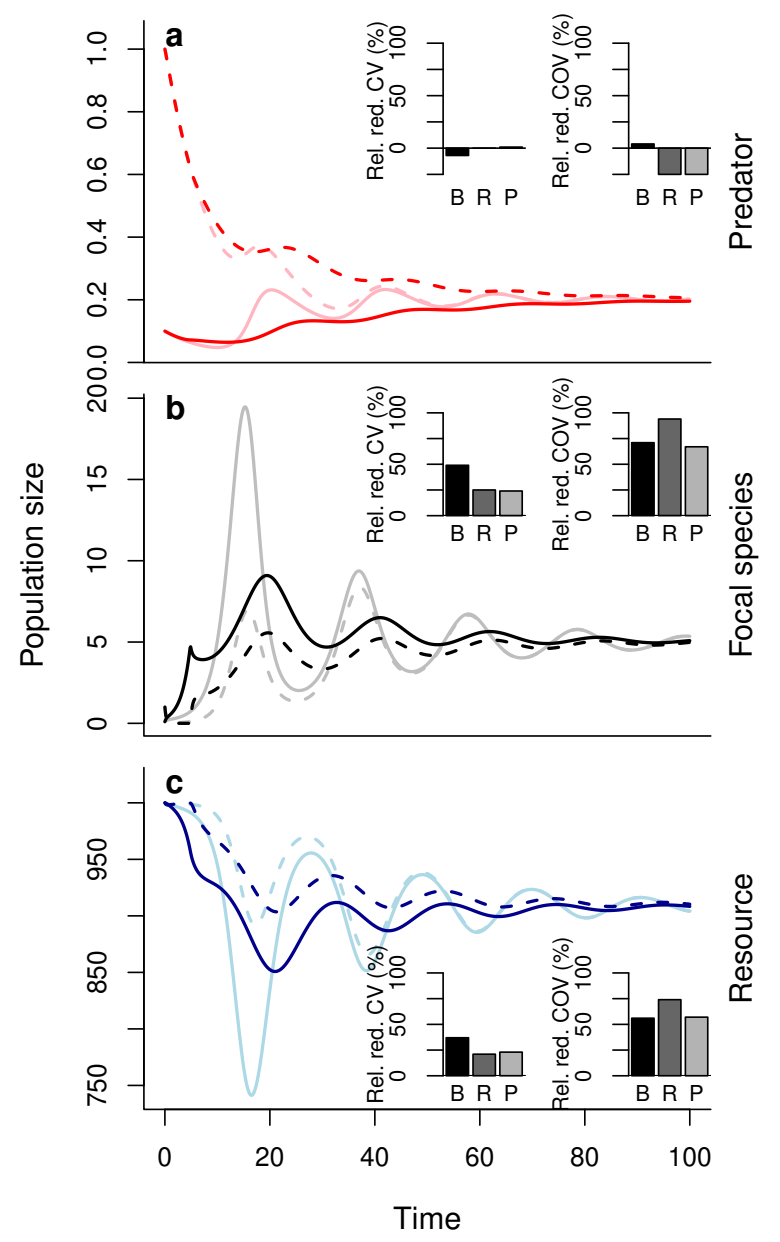

Figure 2: Consequences of CDD for local and regional metacommunity dynamics. We show the dynamics of all three trophic levels (a: top predator in red, $P$; b: focal species in black, $N$; c: resources in blue, $R$ ) in both patches (patch 1: solid lines, patch 2: dashed lines). While the random dispersal (RD; light colours) and context-dependent dispersal (CDD; dark colours) scenarios are characterized by the same model parameters, we compare the specific scenarios in which the $\mathrm{RD}$, respectively $\mathrm{CDD}$, parameters minimize the focal species' population dynamics CV, that is, the most locally stable communities sensu Wang \& Loreau. ${ }^{28}$ The insets show the reduction (Rel. red.) in coefficients of variation (CV) of dynamics within patches, respectively covariance (COV) between patches, under CDD relative to the RD scenario, as well as the differences between scenarios assuming CDD with respect to resources and predators (B), only resources $(\mathrm{R})$ and only predators $(\mathrm{P})$. The strong local effects are due to emigration being simultaneously resource- and predator-dependent. If CDD is only resource- or predator-dependent, local population fluctuations are reduced to a smaller degree, while the reduction in synchrony may be stronger. The RD emigration rate that minimized the focal species $\mathrm{CV}$ was $m_{N}=0.35$. The corresponding CDD thresholds were $T_{R}=956.94$ and $T_{P}=0.12$. Parameter values: $\omega=0.5, R_{0}=1000, e_{N}=0.1, a_{N}=0.01, d_{N}=0.1$, $e_{P}=0.005, a_{P}=4, d_{P}=0.1$. 
Given the general challenges of forecasting ecological dynamics, ${ }^{5,29}$ the absence of a strong interaction between bottom-up and top-down emigration modulators (Fig. 1) has the advantage of making the prediction of ecological metacommunity dynamics potentially easier. ${ }^{30}$ This finding, along with the general and predictable responses of emigration to bottom-up and top-down influences, is encouraging for projecting the dynamics of spatially structured communities into the future. Of course, the dispersal process is more complex than emigration ${ }^{31}$ and future work should integrate all three phases of dispersal. ${ }^{32}$

Our insights could only be gained using our coordinated distributed experimental approach ${ }^{23,24}$ with well defined and unified experimental protocols that allow us to achieve generality beyond a meta-analysis. We here strongly advocate the widespread use of such large collaborative efforts, as they represent a unique possibility to collect high-quality mechanistic data urgently needed for biodiversity forecasting. ${ }^{5}$

In conclusion, our work provides clear insights into the generality of the resource- and predationdependency of the first dispersal phase, emigration. We highlight the potential for far reaching consequences of the multi-causal nature of dispersal, as well as its cascading effects on regional metacommunity dynamics. 


\section{References}

[1] Bonte, D. \& Dahirel, M. Dispersal: a central and independent trait in life history. Oikos 126, $472-479$ (2017).

[2] Hanski, I. A. \& Gaggiotti, O. E. Ecology, Genetics and Evolution of Metapopulations (Academic Press, Inc., 2004).

[3] Vellend, M. Conceptual synthesis in community ecology. Q. Rev. Biol. 85, 183-206 (2010).

[4] Morgan, A. D., Gandon, S. \& Buckling, A. The effect of migration on local adaptation in a coevolving host-parasite system. Nature 437, 253-256 (2005).

[5] Urban, M. C. et al. Improving the forecast for biodiversity under climate change. Science 353, aad8466-1 - aad8466-9 (2016).

[6] Thompson, P. L. \& Gonzalez, A. Dispersal governs the reorganization of ecological networks under environmental change. Nat. Ecol. Evol. 1, 0162 (2017).

[7] Berg, M. P. et al. Adapt or disperse: understanding species persistence in a changing world. Global Change Biol. 16, 587-598 (2010).

[8] Clobert, J., Le Galliard, J. F., Cote, J., Meylan, S. \& Massot, M. Informed dispersal, heterogeneity in animal dispersal syndromes and the dynamics of spatially structured populations. Ecol. Lett. 12, 197-209 (2009).

[9] Matthysen, E. Multicausality of dispersal: a review. In Clobert, J., Baguette, M. \& Benton, T. G. (eds.) Dispersal Ecology and Evolution, 3-12 (Oxford Univ Press, Oxford, UK, 2012).

[10] Legrand, D. et al. Ranking the ecological causes of dispersal in a butterfly. Ecography 38, 822-831 (2015).

[11] Imbert, E. \& Ronce, O. Phenotypic plasticity for dispersal ability in the seed heteromorphic Crepis sancta (Asteraceae). Oikos 93, 126-134 (2001).

[12] Aguillon, S. M. \& Duckworth, R. A. Kin aggression and resource availability influence phenotypedependent dispersal in a passerine bird. Behav. Ecol. Sociobiol. 69, 625-633 (2015).

[13] Matthysen, E. Density-dependent dispersal in birds and mammals. Ecography 28, 403-416 (2005).

[14] Bitume, E. V. et al. Density and genetic relatedness increase dispersal distance in a subsocial organism. Ecol. Lett. 16, 430-437 (2013). 
[15] Bestion, E., Teyssier, A., Aubret, F., Clobert, J. \& Cote, J. Maternal exposure to predator scents: offspring phenotypic adjustment and dispersal. Proc. R. Soc. B-Biol. Sci. 281, 20140701 (2014).

[16] Fronhofer, E. A., Klecka, J., Melián, C. \& Altermatt, F. Condition-dependent movement and dispersal in experimental metacommunities. Ecol. Lett. 18, 954-963 (2015).

[17] Travis, J. M. J., Murrell, D. J. \& Dytham, C. The evolution of density-dependent dispersal. Proc. R. Soc. B-Biol. Sci. 266, 1837-1842 (1999).

[18] Poethke, H. J. \& Hovestadt, T. Evolution of density- and patch-size-dependent dispersal rates. Proc. R. Soc. B-Biol. Sci. 269, 637-645 (2002).

[19] Poethke, H. J., Weisser, W. W. \& Hovestadt, T. Predator-induced dispersal and the evolution of conditional dispersal in correlated environments. Am. Nat. 175, 577-586 (2010).

[20] Amarasekare, P. Spatial dynamics of communities with intraguild predation: The role of dispersal strategies. Am. Nat. 170, 819-831 (2007).

[21] Amarasekare, P. Effect of non-random dispersal strategies on spatial coexistence mechanisms. $J$. Anim. Ecol. 79, 282-293 (2010).

[22] Bowler, D. E. \& Benton, T. G. Causes and consequences of animal dispersal strategies: relating individual behaviour to spatial dynamics. Biol. Rev. 80, 205-225 (2005).

[23] Fraser, L. H. et al. Coordinated distributed experiments: an emerging tool for testing global hypotheses in ecology and environmental science. Front. Ecol. Environ. 11, 147-155 (2013).

[24] Borer, E. T. et al. Finding generality in ecology: a model for globally distributed experiments. Methods Ecol. Evol. 5, 65-73 (2014).

[25] Clobert, J., Baguette, M., Benton, T. G. \& Bullock, J. M. Dispersal Ecology and Evolution (Oxford University Press, Oxford, UK, 2012).

[26] Dell, A. I., Pawar, S. \& Savage, V. M. Temperature dependence of trophic interactions are driven by asymmetry of species responses and foraging strategy. J. Anim. Ecol. 83, 70-84 (2014).

[27] Stevens, V. M. et al. A comparative analysis of dispersal syndromes in terrestrial and semi-terrestrial animals. Ecol. Lett. 17, 1039-1052 (2014).

[28] Wang, S. \& Loreau, M. Ecosystem stability in space: $\alpha, \beta$ and $\gamma$ variability. Ecol. Lett. 17, 891-901 (2014). 
[29] Petchey, O. L. et al. The ecological forecast horizon, and examples of its uses and determinants. Ecol. Lett. 18, 597-611 (2015).

[30] Beckage, B., Gross, L. J. \& Kauffman, S. The limits to prediction in ecological systems. Ecosphere 2, 1-12 (2011).

[31] Jacob, S. et al. Gene flow favours local adaptation under habitat choice in ciliate microcosms. Nat. Ecol. Evol. 1, 1407 (2017).

[32] Travis, J. M. J. et al. Modelling dispersal: an eco-evolutionary framework incorporating emigration, movement, settlement behaviour and the multiple costs involved. Methods Ecol. Evol. 3, 628-641 (2012).

[33] Altermatt, F. et al. Big answers from small worlds: a user's guide for protist microcosms as a model system in ecology and evolution. Methods Ecol. Evol. 6, 218-231 (2015).

[34] Legrand, D. et al. The metatron: an experimental system to study dispersal and metaecosystems for terrestrial organisms. Nat. Methods 9, 828833 (2012).

[35] Ronce, O. How does it feel to be like a rolling stone? Ten questions about dispersal evolution. Annu. Rev. Ecol. Evol. Syst. 38, 231-253 (2007).

[36] Pennekamp, F., Schtickzelle, N. \& Petchey, O. L. Bemovi, software for extracting behavior and morphology from videos, illustrated with analyses of microbes. Ecol. Evol. 5, 2584-2595 (2015).

[37] Burnham, K. P., Anderson, D. R. \& Huyvaert, K. P. Aic model selection and multimodel inference in behavioral ecology: some background, observations, and comparisons. Behav. Ecol. Sociobiol. 65, 23-35 (2011).

[38] McElreath, R. Statistical Rethinking: a Bayesian course with examples in $R$ and Stan (Chapman \& Hall/CRC, 2016).

[39] Metz, J. A. J. \& Gyllenberg, M. How should we define fitness in structured metapopulation models? Including an application to the calculation of evolutionarily stable dispersal strategies. Proc. R. Soc. B-Biol. Sci. 268, 499-508 (2001). 


\section{Acknowledgments}

FA thanks the Swiss National Science Foundation (Grant No. PP00P3_150698). DB and SM thank the FWO (Fonds Wetenschappelijk Onderzoek — grant n. 11T7518N LV). SJ, EL and NS thank UCL and F.R.S.-FNRS; SJ acknowledges a "Move-In Louvain" postdoc position at UCL; NS is Research Associate of F.R.S.-FNRS. DL and MD thank Fyssen Foundation for funding. JDR thanks the FWO (Research Foundation Flanders - grant n. FWO14/ASP/075). FrP was financially supported by Swiss National Science Foundation Grant 31003A_159498. JC was supported by an ANR-12-JSV7-0004-01, by the ERANet BiodivERsA, with the national funder ONEMA, part of the 2012-2013 BiodivERsA call for research proposals and by the French Laboratory of Excellence project "TULIP" (ANR-10-LABX-41). This work was supported by an 'Investissements d'avenir' programme from the Agence Nationale de la recherche number ANR-11-INBS-0001AnaEE-Services.

DL and JC thank Audrey Trochet and Olivier Calvez for their valuable input in experiments involving newts, toads and snakes. DL and JC thank Lucie Raymond for providing butterflies. MD, AA and LM are especially grateful to Christelle Van Gheluwe for running the experiments, and to Maryvonne Charrier for providing Deroceras reticulatum slugs. We thank Mridul Thomas for valuable input on statistical analyses.

\section{Author contributions}

Author names are sorted alphabetically in the respective sections. All authors commented on drafts and have read and approved the final manuscript. More details on individual contributions can be found in the Supplementary Information at the beginning of each specific Supplementary methods section.

Designed the research FA, DB, AC, JC, MD, FDL, EAF, DL, SJ, EL, SM, FrP, NS. This research was designed during a meeting of the dispNet group (https://dispnet.github.io/) organized at UCL by NS and DB.

Performed the experiments FA, AA, SB, DB, JC, MD, FDL, JDR, LdG, EAF, DL, SJ, OK, EL, CJL, LM, FM, SM, FeP, FrP, NS, LT, AV, LW. More information can be found in the Supplementary Information.

Analysed the experimental data JC, EAF

Designed and analysed the model EAF 
Drafted the manuscript EAF

\section{Competing interests statement}

The authors declare no competing interests. 


\section{Material and methods}

\section{Study organisms}

We used 21 focal study species (Armadillidium vulgare (license for the predator: 09-2016-02 and 2012-10 DREAL), Chilomonas sp., Colpidium sp., Cornu aspersum, Cryptomonas sp., Deroceras reticulatum, Dexiostoma sp., Dikerogammarus villosus, Gammarus fossarum, Lissotriton helveticus (license: 09-201602), Paramecium caudatum, Phoxinus phoxinus (license: E-2016-130), Pieris brassicae (license for the predator: 09-2016-02), Pirata latitans (license for the predator: 2012-10 DREAL), Platycnemis pennipes (license for the predator: 09-2016-02), Pteronemobius heydenii (license for the predator: 09-2016-02 and 2012-10 DREAL), Tetrahymena elliotti, Tetrahymena pyriformis, Tetrahymena thermophila, Tetranychus urticae, Zootoca vivipara (license: 2012-10 DREAL)), including aquatic, terrestrial and aerially dispersing taxa of protists, algae, arthropods, molluscs and vertebrates. Resources and predators of these focal species were chosen based on known natural co-occurrences to allow for the possibility of a common evolutionary history (see Supplementary Information for details).

\section{Experimental setup and treatments}

Experiments across all study species followed the same general experimental procedure. We used experimental two-patch systems adapted to each study species (for example, species-specific patch sizes, corridor size and positions) in order for experimental populations to reflect naturally occurring densities and living conditions. Experimental conditions therefore ranged from connected microcosms ${ }^{33}$ to semi-natural connected mesocosms (the Metatron ${ }^{34}$ ).

Importantly, all experimental metacommunities were characterized by the presence of a 'hostile matrix' connecting the patches, which ensured that inter-patch relocation was indeed dispersal, ${ }^{22,25,35}$ that is, a change of habitat with potential consequences for gene flow, and not routine foraging movement (see the Supplementary Information for details).

We applied a full factorial design crossing two levels of resource availability (RA) and predation risk (PRED). Resources were ad libitum ('standard' condition; standard RA) or seriously limiting (low RA). Predation risk (PRED) was represented by the presence (yes PRED) or absence of cues (no PRED) belonging to a natural and relevant (i.e., shared evolutionary history) predator of the focal species. Predator cues could be chemical, visual and auditory, depending on the biology of the focal species. We manipulated predator cues instead of the physical presence of predators in order to avoid concurrent effects on population dynamics. The treatments were always applied to one patch ('origin') that was initially populated by similar densities of individuals of the focal species for each treatment. The second patch ('target') always had reference conditions (standard resources, no predator cues) and was initially 
empty.

After placing a population of individuals in the 'origin' patch, treatments were applied at the beginning of an acclimation phase which took approximately one quarter of the time of the subsequent dispersal phase. During the acclimation phase no dispersal was possible. The absolute time of the acclimation and dispersal phases were adapted depending on the focal species (see Supplementary Information). All treatments were replicated 5 times, with the exception of few species where replication was lower $(2$ replicates for Pieris brassicae and Platycnemis pennipes respectively; 4 replicates for Zootoca vivipara) or higher (6 replicates for Armadillidium vulgare, Lissotriton helveticus, Phoxinus phoxinus, Pirata latitans and the protists except Tetryhamena thermophila; 9 and 10 replicates for Dikerogammarus villosus and Gammarus fossarum, respectively; 8 replicates for Pteronemobius heydenii) due to experimental constraints (for details see Supplementary Table 1). For some species, the experimental design included a block, which always included replicates of all treatments and was accounted for in the statistical analysis (see below). The coordinated distributed experiment on the 21 focal species was carried out in 7 different laboratories across Europe (see Supplementary Table 1).

\section{Data collection}

Data on dispersal, more specifically emigration, that is, the number of residents (individuals in the patch of origin at the end of the experiment) and dispersers (individuals that had left their patch of origin and were in the target patch at the end of the experiment) after the dispersal phase in each replicate, were either collected using video recording and analysis ${ }^{36}$ or by direct observation. Using data from further analyses or literature surveys (specified in the Supplementary Information), we collected species specific information for the focal species, resources and predators including: movement, space use, feeding strategy, body size, predator specialization and focal species escape strategies. The latter information was either used directly or in relevant focal species to predator ratios as potential explanatory variables for understanding the modulators of resource and predator impacts on emigration (see Supplementary Table 1).

\section{Statistical analysis}

All statistical analyses were performed using the R Language and Environment for Statistical Computing (version 3.4.4) and occurred in two steps. We analysed overall treatment effects on all species together using generalized linear mixed effects models (GLMM) on proportion counts of residents and dispersers (aggregate binomial regression; binomial error structure with logit link function; 'glmer' function of the 'Ime4' package using the 'bobyqa' optimizer). As random effects we included experimental block within 
species within taxon. We used taxon as a random effect to account for potential phylogenetic nonindependence and included the levels 'protists', 'algae', 'arthropods', 'molluscs' and 'vertebrates' (see Supplementary Table 1). We further included the laboratory in which the experiment was performed as a random effect in order to account for potential experimenter effects. Overdispersion was accounted for by additionally including an observation level random effect. Model selection was performed on all models from the full model which included an interaction between resource availability and predation risk to the intercept model using AICc. ${ }^{37}$ Besides identifying the most parsimonious model, we also provide information on relative variable importance, which is the sum of AICc weights of models in which the variable of interest occurs.

In an exploratory, post-hoc analysis, species-specific models were used to extract log odds ratios. Subsequently, these log odds ratios were used to determine species-specific modulators of the global CDD response. Model structure for obtaining log odds ratios (logORs) of both bottom-up (resource availability) and top-down (predation risk) effects was analogous to the global analysis described above. However, the only potential random effect at the species level was 'block'. In case the specific experiment did not include a block we used a GLM and potential overdispersion was accounted for by using a 'quasibinomial' error structure. We only modelled an additive effect of resource availability and predation risk, as the global analysis suggested the absence of an interaction (see results). We nevertheless provide the analysis of the species level effects based on models including the interaction between the two explanatory variables in the Supplementary Tables 7-12. For the subsequent analyses, one protist species (Chilomonas sp.) was excluded, as the $\log \mathrm{OR}$ and the associated errors were meaningless due to zero emigration in the reference treatment (standard resources, no predation).

The statistical analysis of the species level $\log$ ORs and potential explanatory variables was executed in a meta-analysis framework in order to account for the uncertainty associated with each species specific $\log \mathrm{OR}$ ('rma.mv' function of the 'metafor' package). Again, 'taxon' and 'laboratory' were included as random effects. Model selection using AICc was performed on the additive models including all possible combinations of explanatory variables, which can be found in Supplementary Table 1). Specifically, we used 'focal species ID', 'relevant taxon', 'dispersal mode', 'focal species feeding strategy' and 'log(focal body size)' for the effect of resource limitation and 'focal species ID', 'relevant taxon', 'dispersal mode', 'rel. space use', 'predator mobility', 'predator feeding strategy', 'predator specialization', 'escape strategy', ' $\log ($ focal body size)' and 'log body size ratio' for the effect of predation. For further information see Supplementary Table 1. We included 'focal species ID' to test whether the responses were truly species specific, that is, varied idiosyncratically between species, or were more readily explained by other explanatory variables. For visualization, model predictions were averaged using AICc model weights as proportions. $^{38}$ 


\section{A simple two-patch food-chain model with CDD}

To illustrate the consequences of context-dependent, more precisely resource- and predation-dependent emigration, we explored the dynamics of a simple, two-patch food-chain model that captures the essence of our experimental setting. The basal resource $(R)$ is abiotic and flows in and out of the system at a given rate $(\omega)$. The focal species $(N)$ feeds upon this resource and is itself subject to predation by a top predator $(P)$. For simplicity, we assume that both consumers follow a linear, that is type I, functional response (feeding rate $a$ ) and that only the focal species is able to disperse (emigration rate $m_{N}$; see Supplementary Figures $4-5$ for an exploration of the consequences of predator dispersal). The dynamics of this food chain in patch $i$ are given by:

$$
\begin{aligned}
\frac{d P_{i}}{d t} & =e_{P} a_{P} N_{i} P_{i}-d_{P} P_{i} \\
\frac{d N_{i}}{d t} & =e_{N} a_{N} R_{i} N_{i}-d_{N} N_{i}-a_{P} P_{i} N_{i}+m_{N}\left(N_{j}-N_{i}\right) \\
\frac{d R_{i}}{d t} & =\omega R_{0}-\omega R_{i}-a_{N} N_{i} R_{i}
\end{aligned}
$$

where $e$ is the assimilation coefficient, $R_{0}$ the resource concentration flowing into the system. The subscripts either indicate the patch $(i, j)$ or whether the consumer parameters describe the focal species $(N)$ or the top predator $(P)$.

We compared the dynamics of this two-patch food-chain model with random dispersal (RD) and context-dependent dispersal (CDD). In the earlier scenario, $m_{N}$ is an unconditional rate. For CDD, we assume that the emigration reaction norm is a step function as derived by Metz \& Gyllenberg. ${ }^{39}$ The probability to disperse in the latter scenario will be zero if resources are above a threshold resource density and one if they are below. Simultaneously, the emigration rate will be zero if predators are below a threshold predator density and one if they are above. In summary, we assume negative resource-dependent emigration and positive predator-dependent emigration, as we found experimentally.

While the RD and CDD scenarios we contrast are characterized by the same model parameters, we compare the specific scenarios in which the RD and CDD parameters, respectively, minimize the focal species population dynamics coefficient of variation $(\mathrm{CV})$, as a proxy for local population stability. ${ }^{28}$ Alternatively, we compare RD and CDD scenarios that have the same emigration rates as measured at the end of the analysed time series (see Supplementary Figure 3). In analogy to Wang \& Loreau, ${ }^{28}$ we use temporal coefficients of variation within local communities as well as covariances between communities as proxies for (meta)community stability.

The results we report here should be understood as an illustration of potential consequences of CDD. 
Although based on a sound mathematical framework (Eqs. 1a-c) and accompanied by a sensitivity analysis (Supplementary Tables 13-14 and Supplementary Figures $3-5$ ), the results are a snapshot of possible dynamics as a full analysis of the model is beyond the scope of this manuscript.

\section{Data and code availability}

The dataset and computer code generated and analysed during the current study are available in the Zenodo repository, DOI: 10.5281/zenodo.1344579 . 


\section{Supplementary Information}

Emanuel A. Fronhofer, Delphine Legrand, Florian Altermatt, Armelle Ansart, Simon

Blanchet, Dries Bonte, Alexis Chaine, Maxime Dahirel, Frederik De Laender, Jonathan

De Raedt, Lucie di Gesu, Staffan Jacob, Oliver Kaltz, Estelle Laurent, Chelsea J. Little, Luc Madec, Florent Manzi, Stefano Masier, Felix Pellerin, Frank Pennekamp, Nicolas

Schtickzelle, Lieven Therry, Alexandre Vong, Laurane Winandy and Julien Cote:

\section{Bottom-up and top-down control of dispersal across major organismal groups}




\section{Supplementary Methods}

\section{Common woodlouse - Armadillidium vulgare, Marsh cricket - Pteronemo- bius heydenii, Wolf spider — Pirata latitans and Palmate newt — Lissotriton helveticus}

Authors Delphine Legrand, Alexandre Vong, Laurane Winandy and Julien Cote

License 2012-10 DREAL (common lizard); 09-2016-02 (common toad); 09-2016-02 (palmate newt)

\section{Study organisms and predators}

Armadillidium vulgare The common woodlouse (Armadillidium vulgare, Latreille 1804) is a widespread European woodlouse species (average body length: $18 \mathrm{~mm}$ ). In this experiment, we used 236 woodlice captured in the Metatron ${ }^{1}$ and maintained in small tanks $(22 \mathrm{~L}, 39$ x 28 x $28 \mathrm{~cm})$. Tanks contained $10 \mathrm{~cm}$ of soil litter, 3 egg boxes used as refuges, two small dishes for water and a regular addition of decaying vegetables (apple, grass, potatoes and carrots). ${ }^{2}$ As we caught woodlice in semi-natural enclosures, they had never been exposed to predator cues (see below).

The common lizard (Zootoca vivipara; adult snout-vent length: males, 40-60 mm; females, 45-75 mm) and the common toad (Bufo bufo; body length: males, approx. $69 \mathrm{~mm}$; females, approx. $93 \mathrm{~mm})^{3}$ were used as our model predator. Both species are generalist feeders preying upon various arthropods species including woodlice. ${ }^{3,4}$ Lizards were raised in cattle tanks as described in the common lizard protocol and toads were captured in the Metatron.

We estimated that woodlice have smaller home ranges than lizards and toads $\left(<0.3 \mathrm{~m}^{2}\right.$ for woodlice estimated from another isopod species, ${ }^{5}<1200 \mathrm{~m}^{2}$ for common lizards, ${ }^{6} 8000-28000 \mathrm{~m}^{2}$ for common toads $\left.^{7}\right)$. Mobility, estimated as sprint speed, was higher for lizards ${ }^{8,9}$ and toads ${ }^{10}$ than for woodlice ${ }^{11}$ (lizard: $30-60 \mathrm{~cm} \mathrm{~s}^{-1}$, common toad: approx. $30 \mathrm{cms}^{-1}$, woodlouse: $10 \mathrm{~cm} \mathrm{~s}^{-1}$ ). On top of dispersal, woodlice have several other anti-predator strategies, including aggregation, sheltering, armour, alarm cue, and repellent chemicals. ${ }^{12}$

Pteronemobius heydenii The Marsh cricket (Pteronemobius heydenii, Fischer 1853) is a small, omnivorous cricket with a brown-black body (males: 5-7 mm, females 6-8 mm) found in humid habitats throughout Eurasia. ${ }^{13,14}$ We used 311 crickets captured in the Metatron ${ }^{1}$ and maintained in small tanks (22 L, $39 \times 28 \times 28 \mathrm{~cm})$. Tanks contained $10 \mathrm{~cm}$ of soil litter, 3 egg boxes used as refuges, two small dishes for water and a regular addition of fresh vegetables (apple, grass, potatoes and carrots) after checking that the species effectively fed on them. As we caught crickets in semi-natural enclosures, they had never been exposed to predator cues (see below). 
The common lizard (Zootoca vivipara) and the common toad (Bufo bufo) were used as our model predator. Both species are generalist feeders preying upon various arthropods species including crickets. ${ }^{3,4,15}$ Lizards were raised in cattle tanks as described in the common lizard protocol and toads were captured in the Metatron.

We estimated that crickets had smaller home ranges than lizards and toads $\left(<10 \mathrm{~m}^{2}\right.$ for crickets, roughly estimated from a net squared displacement of a similar sized cricket species, ${ }^{16}<1200 \mathrm{~m}^{2}$ for common lizards, ${ }^{6} 8000-28000 \mathrm{~m}^{2}$ for common toads $\left.{ }^{7}\right)$. Mobility, estimated as sprint speed, was similar for the 3 species (lizards: $30-60 \mathrm{~cm} \mathrm{~s}^{-1}, 8,9$ toad: approx. $30 \mathrm{cms}^{-1},{ }^{10}$ crickets: $25 \mathrm{~cm} \mathrm{~s}^{-1}$, we estimated the escape speed of crickets using the escape success-predator velocity relationship described in $^{17}$ ).

Pirata latitans The wolf spider Pirata latitans (Blackwall, 1841) is a European species of the family Lycosidae (body length: $3.5-5 \mathrm{~mm}$ ) occurring in wet and marshy areas. Wolf spiders are generalists and active hunters. In this experiment, we used 257 spider captured in the Metatron ${ }^{1}$ and maintained in small tanks $(5 \mathrm{~L}, 28 \times 20 \times 14 \mathrm{~cm})$. Tanks contained $5 \mathrm{~cm}$ of soil litter, tiles used as refuges, a regular addition of cricket (Acheta domestica), and were sprayed with water twice a day. As we caught spiders in semi-natural enclosures, they had never been exposed to predator cues (see below).

The common lizard (Zootoca vivipara) was used as our model predator. Common lizards are generalists and active feeders preying upon various arthropods species with a noticeable preference for spiders. ${ }^{4}$ Lizards were raised in cattle tanks as described in the common lizard methods section (see below).

We estimated the prey and predator species to have home ranges of similar magnitudes $\left(300-900 \mathrm{~m}^{2}\right.$ for other wolf spiders, ${ }^{18}<1200 \mathrm{~m}^{2}$ for common lizards ${ }^{6}$ ). The mobility, estimated as sprint speed, was also similar (lizards: $30-60 \mathrm{~cm} \mathrm{~s}^{-1}, 8,920 \mathrm{~cm} \mathrm{~s}^{-1}$ for spiders of the same genus ${ }^{19}$ ).

Lissotriton helveticus The palmate newt (Lissotriton helvetucus, Razoumovsky, 1789) is a small European newt species (adult length: males, up to $85 \mathrm{~mm}$; females, $95 \mathrm{~mm}$ ) that often aggregates in large groups. ${ }^{20}$ In this experiment, we used 216 small newts $(17.8 \pm 2.6 \mathrm{~mm} \mathrm{SE})$ in the terrestrial phase captured in the Metatron ${ }^{1}$ and maintained in 4 terrariums (35 x $17.5 \times 22.5 \mathrm{~cm}, 54$ individuals per terrarium). Terrariums contained $5 \mathrm{~cm}$ of soil litter covered with mosses, 2 pieces of egg carton used as refuges, two small dishes for water and a regular addition of bloodworms. As we caught newts in semi-natural enclosures, they had never been exposed to predator cues (see below).

The grass snakes (Natrix natrix) were used as our model predator. Grass snakes are active and generalist feeders, preying upon amphibians, fish, small mammals, reptiles and birds. ${ }^{21-23}$ Grass snakes forage on newts mainly in their aquatic phase. We used several snakes maintained in a reptile zoo.

We estimated that newts had similar home ranges as snakes $\left(<50000 \mathrm{~m}^{2}\right.$ for newts ${ }^{24,25} 12000-$ $36000 \mathrm{~m}^{2}$ for grass snakes ${ }^{26,27}$ ), while the mobility, estimated as sprint speed, was higher for snakes than for newts (newts: $<10 \mathrm{cms}^{-1}$, in another newt genus, ${ }^{28,29}$ snakes: $30-60 \mathrm{~cm} \mathrm{~s}^{-1},{ }^{21,30}$ ). On top 
of dispersal, newts can exhibit a postural defence exposing ventral orange colouration. ${ }^{31}$ During this posture there is a release of skin secretion (tetrodotoxin) making them inedible to predators. ${ }^{32}$

\section{Experimental setup}

For the four species, we used 8 two-patch systems placed in a greenhouse with controlled temperature $\left(16-25^{\circ} \mathrm{C}\right)$. Each system was made of two $130 \mathrm{~L}$ plastic containers $(78 \times 56 \times 43 \mathrm{~cm})$ connected by a circuitous plastic pipe (diameter: $10 \mathrm{~cm}$, total length: $4.4 \mathrm{~m}$ ) on the upper section of the container. The origin patch was filled with soil litter providing access to the corridor while we only added a thin layer of soil in corridors and target patch. To go from the origin to the target patch, individuals had to enter this narrow corridor and fall into the target patch.

\section{Treatments}

We created 24 populations of woodlice, spiders and newts and 32 populations of crickets. Populations were made of 8-11 individuals (woodlice: approx. $9.83 \pm 0.10$ SE, crickets: approx. $9.71 \pm 0.11$ SE, spiders: approx. $9.83 \pm 0.10$ SE, newts: approx. $8.23 \pm 0.29 \mathrm{SE}$ ). We used a 2 x 2 factorial design, crossing resource availability (RA) and predation risk (PRED) with two levels each, resulting in six replicates of each combination of treatments for woodlice, spiders and newts and eight replicates for crickets. Species were tested separately in 3 blocks for woodlice, spiders and newts and 4 blocks for crickets (woodlice: from September 10th to October 13th 2016, crickets: from September 26th to October 20th 2016, spiders: from July 29h to September 6th 2016, newts: from November 23rd to December 15th 2016). The RA and PRED treatments were applied before releasing individuals of each species for a 24 hours acclimation phase with connections between patches closed. After 24 hours, connections were opened and we monitored dispersal movements as described below.

Resource availability RA included two treatments: a low and standard RA treatment with a large difference of food availability between treatments. For woodlice and crickets, the resources were manipulated through the addition of decayed vegetables (half a potato, half a carrot, half an apple and a hand-full of grass) in the high resource treatment and only a very small piece of vegetable and 2 pieces of grass in the low resource treatment. For spiders, the resources were manipulated through the addition of 100 crickets in the standard RA treatment and 25 in the low RA treatment. For newts, the resources were manipulated through the addition of approx. 90 bloodworms in the standard RA treatment and approx. 10 in the low RA.

Predator cues: Armadillidium vulgare, Pteronemobius heydenii and Pirata latitans Lizards were housed in individual terrariums containing $3 \mathrm{~cm}$ of soil, a shelter (a piece of eggs carton), a water dish, and a piece of absorbent paper to collect odours serving as predator cues. In one corner 
of the terrarium, ultraviolet and incandescent lamps provided light and heat for thermoregulation from 9:00 to 12:00 and from 14:00 to 17:00. Lizards were fed daily with 1 cricket (Acheta domestica). We used a mix of several absorbent papers belonging to several lizard terraria. Four toads were maintained all together in a large plastic tank $(130 \mathrm{~L}, 78 \times 56 \times 43 \mathrm{~cm})$ with rocks, water, soil and absorbent paper. Tanks were watered daily and abundantly to maintain enough humidity. Before releasing individuals, two-patch systems with predator cues received a piece of lizard paper mixes, whereas control treatments received a piece of odour free paper collected from vacant terraria maintained under the same conditions than inhabited terraria. Woodlice and cricket systems additionally received a piece of toad paper. When removing a paper from predator or predator-free containers (for lizards and toads), we added a new piece of paper for future experimental blocks. The protocol to collect lizard olfactory cues is a standard protocol already used several times to elicit social reactions in lizards. ${ }^{33,34}$ For woodlice, we also added two individuals that were crushed in a tube in each predator treatment as alarm cues of predation and an empty tube in no PRED treatments. We did so to insure their perception of predation risk as woodlice are known to react to predator chemical cues ${ }^{2}$ and to alarm cues ${ }^{12}$.

Predator cues: Lissotriton helveticus Snakes were housed in a reptile zoo (https://www.lafermedesreptiles.fr/). For each population, we used wood chips maintained in snake boxes. Before releasing newts, origin patches of yes PRED treatments received a small proportion of wood chips from a snake box, whereas no PRED treatments received wood chips free of odour. A similar protocol to collect snake olfactory cues has been shown to be efficient in eliciting antipredator reactions in terrestrial salamander species. ${ }^{35-37}$ While newts' responses to predator chemical cues have been mostly studied in the aquatic phase, ${ }^{38}$ newts in the terrestrial phase are known to use olfactory cues to select habitats. ${ }^{39}$

\section{Dispersal and data collection}

After the opening of corridors, we monitored dispersal daily for 15, 5, 15 and 1 days for woodlice, crickets, spiders and newts, respectively. To do so, we captured dispersers in the target patches without disturbing the origin patches. At the end of the dispersal assay, we captured all individuals of each species in origin and target patches. We recaptured 114 woodlice, 266 crickets, 153 spiders and 177 newts. We only used recaptured ones in our analysis to prevent confounding dispersal with recapture probability and survival. 


\section{Protists - Chilomonas sp., Colpidium sp., Tetrahymena pyriformis and Desxiostoma sp.}

Authors Emanuel A. Fronhofer, Frank Pennekamp and Florian Altermatt

\section{Study organisms and predators}

Protist microcosms have a long tradition of being used in ecological and evolutionary studies. ${ }^{40}$ We here used a set of four predator-prey species pairs cultured in protist pellet medium $\left(0.46 \mathrm{~g} \mathrm{~L}^{-1}\right.$; Carolina Biological Supply) with a mix of three bacterial species as a basal resource (Serratia fonticola, Bacillus subtilis and Brevibacillus brevis). The four species pairs included the focal species Chilomonas sp. (body size, as length along the major body axis: $29.7 \mu \mathrm{m}$; swimming speed, as mean net speed: $4.72 \mu \mathrm{ms}^{-1}$ ) with Blepharisma sp. (body size: $91.61 \mu \mathrm{m}$; swimming speed: $6.94 \mathrm{\mu m} \mathrm{s}^{-1}$ ) as a more mobile predator, the focal species Colpidium sp. (body size: $75.4 \mu \mathrm{m}$; swimming speed: $15.59 \mathrm{\mu m} \mathrm{s}^{-1}$ ) with the sessile predator species Stentor sp. (body size: approx. $800 \mu \mathrm{m}$ ), Tetrahymena pyrformis (body size: $37.83 \mu \mathrm{m}$; swimming speed: $9.22 \mu \mathrm{m} \mathrm{s}^{-1}$ ) as a focal species with Cephalodella sp. (body size: approx. $300 \mu \mathrm{m}$; swimming speed: approx. $10 \mathrm{\mu m} \mathrm{s}^{-1}$ ), a rotifer, as a slightly more mobile species and finally the focal species Dexiostoma sp. (body size: $36.31 \mu \mathrm{m}$; swimming speed: $13.16 \mu \mathrm{ms}^{-1}$ ) with the less mobile predator Dileptus sp. (body

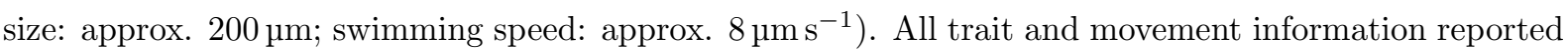
here was measured during the experiments. All protist cultures were originally obtained from Carolina Biological Supply. All focal species graze on the bacterial resource and all predators are generalists and exhibit an active capture strategy, except for Stentor sp. which is sessile and therefore a sit-and-wait predator.

\section{Experimental setup}

We used replicated two-patch systems consisting of two $20 \mathrm{~mL}$ vials (Sarstedt) connected by silicone tubing (VWR) as previously used in ${ }^{41}$ or. ${ }^{42}$ While the inside diameter of the connecting tube was fixed to $4 \mathrm{~mm}$, the length was adjusted to control for different dispersal capacities among focal species: Chilomonas sp. and Dexiostoma sp.: $7 \mathrm{~cm}$; Colpidium sp. and T. pyriformis: $3.5 \mathrm{~cm}$.

At the beginning of the experiment the tube connecting the two patches was filled with water instead of protist medium as a 'hostile matrix' preventing cell growth and hence reproduction and clamps were used to close the connection. After a $1 \mathrm{~h}$ acclimation phase, the clamps were removed and dispersal was allowed for $4 \mathrm{~h}$. All predator-prey systems were replicated six times.

\section{Treatments}

The composition of the origin patch depended on the treatment combination which is described below (total volume: $15 \mathrm{~mL}$ ). By contrast, the target patches all received $13 \mathrm{~mL}$ of fresh, sterile medium as 
well as $2 \mathrm{~mL}$ bacterial culture at equilibrium (approx. 1 week old culture) as food resources for the focal species.

Resource availability Resource availability (RA) in the origin patch was either equal to the target patch (standard RA), that is, the patch received $2 \mathrm{~mL}$ bacteria at equilibrium (standard RA), or severely limited by not providing any bacterial food (low RA: $2 \mathrm{~mL}$ water instead of bacteria). To these $2 \mathrm{~mL}$ we added $3 \mathrm{~mL}$ of a concentrated culture of the focal species. In order to obtain this concentrated culture, we used $15 \mathrm{~mL}$ of a culture of the focal species at equilibrium, centrifuged it (Sigma 3-16PK centrifuge; $5 \mathrm{~min}$ at $4500 \mathrm{rpm}$ ) and washed out the pellet containing the protist cells with $3 \mathrm{~mL}$ fresh and sterile medium.

Predator cues The remaining $10 \mathrm{~mL}$ of the origin patch consisted either of fresh and sterile medium (no PRED) or sterile filtered medium (filter pore size: $0.2 \mu \mathrm{m}$ ) from the respective predator culture at equilibrium (yes PRED). The sterile filtered medium from the predator culture contained no bacterial resources but all chemical compounds secreted by the predators which may be used as a predator cue by the focal species. This procedure guaranteed that, regardless the concentration of resources or predator cues (see below) the focal population in the starting patch was always at equilibrium, as the total volume was again $15 \mathrm{~mL}$.

\section{Dispersal and data collection}

After $1 \mathrm{~h}$ acclimation phase and $4 \mathrm{~h}$ dispersal we closed the clamps and took $20 \mathrm{~s}$ videos in origin and target patches using a Leica M205 C stereomicroscope at a 16-fold magnification and a Hamamatsu Orca Flash 4 video camera (imaged volume: $34.4 \mu \mathrm{L}$; height: $0.5 \mathrm{~mm}$ ). Population densities of residents and dispersers were obtained using video analysis and the 'BEMOVI' package for the R Language and Environment for Statistical Computing. ${ }^{43}$ For specific settings see GitHub (https://github.com/efronhofer/analysis script bemovi). 


\section{Garden snail - Cornu aspersum}

Authors Maxime Dahirel, Armelle Ansart and Luc Madec

\section{Study organism and predator}

Cornu aspersum (Müller; Gastropoda, family Helicidae) is a medium-sized land snail common throughout Western Europe. ${ }^{44}$ In May 2016, we collected individuals by hand in suburban populations in Pacé, France $\left(48^{\circ} 9^{\prime} \mathrm{N}, 1^{\circ} 47^{\prime} \mathrm{W}\right)$. Although subadults are overall more dispersive, ${ }^{45}$ we selected only adults and old subadults (snails with diameter greater $25 \mathrm{~mm}$ ) for our experiments, to limit the influence of variation in internal developmental stage. Snails were individually marked with felt-tip paint markers and maintained under controlled conditions $\left(20^{\circ} \mathrm{C} \pm 1^{\circ} \mathrm{C} ; 16 \mathrm{~L}\right.$ : $\left.8 \mathrm{D}\right)$. They were housed by random groups of 10 individuals in $30 \times 30 \times 8 \mathrm{~cm}$ polyethylene boxes with $1 \mathrm{~cm}$ of moist soil at the bottom. This density is well within the range of naturally observed densities near shelters, and below the thresholds at which many negative effects of crowding appear under controlled conditions. ${ }^{46}$ Snails were fed ad libitum with commercial snail food (cereal flour supplemented with calcium, Hélinove, Le Boupère, France) placed on a Petri dish at the centre of the box.

Larvae of the European common glow-worm, Lampyris noctiluca (Linnaeus) are specialist predators of soft bodied invertebrates, especially land snails and slugs. ${ }^{47}$ They are one of the few insect species recorded to predate Cornu aspersum snails, and seem to be able to follow snail mucus trails to reach their prey. ${ }^{47}$ Crawling glow-worm larvae are about twice as fast on average than crawling garden snails (roughly $4-6 \mathrm{~cm} \mathrm{~min}^{-1}$ versus $2-3 \mathrm{~cm} \mathrm{~min}^{-1},{ }^{48,49}$ ). We collected glow-worm larvae by hand in April 2016 in Arçais, France $\left(46^{\circ} 17^{\prime} 13^{\prime \prime} \mathrm{N}, 0^{\circ} 40^{\prime} 3^{\prime \prime} \mathrm{W}\right)$. Due to difficulties in maintaining them in controlled conditions, they were killed within a week of capture, and we used cuticular extracts as predator cues (see below for details on the killing and extraction protocol).

\section{Experimental setup}

Two-patch systems were built inside 30 x $180 \mathrm{~cm}$ plastic forcing tunnels (height: $20 \mathrm{~cm}$ ) placed in a tiled 4 x $4 \mathrm{~m}$ room (mean temperature $20^{\circ} \mathrm{C} ; 16 \mathrm{~L}$ : 8D). Tunnels were duct-taped to the floor in order to prevent escapes. Boxes in which snails were maintained (see above) were placed at one extremity of each tunnel to serve as origin patches; similar boxes, but without snails, were placed on the opposite side to serve as target patches. The between-patch matrix was left empty and dry. With this setup, the between-patch distance was $120 \mathrm{~cm}$; previous observations showed that non-dispersal exploratory movements nearly always spanned distances $<1 \mathrm{~m}$ (M. Dahirel, unpublished data). 


\section{Treatments}

One box of ten snails was released per two-patch system, the box itself serving as the origin patch. We tested 5 systems per combination of predator cue presence (PRED, yes/no) and food restriction (RA, standard/low) treatments, so 20 systems and 200 snails overall (see below for details on the implementation of the treatments). Due to logistical constraints, systems were tested in two blocks of ten (start dates: June 9 and 13, 2016); all four possible treatments were roughly equally distributed between these two blocks (two replicates of each combination of treatments in each block, plus 1 replicate low RA no PRED in block 1 and 1 replicate low RA yes PRED in block 2).

Twenty-four hours before release in the two-patch systems, the original feeders in each box were removed. They were replaced by new Petri dishes, surrounded with a $3 \mathrm{~cm}$ wide ring of Whatman paper with or without predator cues depending on the treatment (see below). Snail food was also added to the new feeders based on treatments (see below). After these 24 hours of acclimation, the origin patch box was placed in a randomly selected system, opened, and snails were left free to disperse for 3 days. Whatman papers were left in place, feeders in the origin patch were refilled with food for 3 days (actual quantity depended on treatment), and feeders in the target patch were filled with enough food to sustain all snails for 3 days.

Resource availability During dispersal trials, snails were fed the same cereal flour they were exposed to during maintenance. Previous tests ${ }^{45}$ and new preliminary experiments showed that snails consume on average $0.4 \mathrm{~g}$ of flour per day when alone and provided with ad libitum food, independently of snail size. Origin patches in the standard RA treatment, and target patches in all treatments, were provided with $8 \mathrm{~g}$ of snail food per day, i.e. twice the quantity needed to sustain 10 snails based on the above result. It is difficult to evaluate the effect of a short-term food restriction on Cornu aspersum, as this species readily enters dormancy; we therefore used the same food restriction as for the land slug Deroceras reticulatum, namely $-90 \%$. Origin patches in the low RA treatment were thus provided with $0.4 \mathrm{~g}$ of snail food per day, i.e. $10 \%$ of the quantity consumed by 10 snails.

Predator cues Cornu aspersum, like most land gastropods, has poor vision and audition, and relies primarily on olfaction to apprehend its environment. ${ }^{50}$ Cuticular extracts of the predator Lampyris noctiluca were extracted following a published protocol. ${ }^{51}$ Briefly, beetle larvae that had been starved for $48 \mathrm{~h}$ to remove digestive residues were placed in a refrigerator $\left(4^{\circ} \mathrm{C}\right)$ for $4 \mathrm{~h}$. This aimed to induce torpor, avoiding the release of defensive secretions during freezing. Larvae were then transferred to a freezer $\left(-20^{\circ} \mathrm{C}\right)$ for $24 \mathrm{~h}$. Dead frozen beetles were then placed in a new glass vial filled with $20 \mathrm{~mL}$ of pure ethanol per gram of live weight. This solution was stored at $4{ }^{\circ} \mathrm{C}$ and gently shaken twice a week. This solution was used two weeks after its creation. Pure ethanol stored under the same conditions was 
used as a control solution. These solutions were then presented to snails using pieces of Whatman paper, with $5 \mu \mathrm{L}$ of solution per $\mathrm{cm}^{2}$ of paper, after leaving paper strips at room temperature for 15 minutes to allow for evaporation of the excess ethanol. To confirm the validity of this extraction protocol and the efficiency of the predatory cue, we placed 12 Cornu aspersum snails in individual $9 \times 6 \times 5 \mathrm{~cm}$ boxes with the bottom lined with two strips of Whatman paper, one side with the predatory cues solution, one side with the control solution. After one hour, all snails had chosen a side, and $83.3 \%$ (10 out of 12) were on the "control" side $\left(\chi^{2}=5.33, p=0.02\right)$.

\section{Data collection}

The number of dispersing (i.e., in the target patch) and non-dispersing (in the origin patch) snails in each system was recorded once after 3 days. 


\section{Cryptomonas sp.}

Authors Jonathan De Raedt and Frederik De Laender

\section{Study organism}

Here, we used Cryptomonas sp. strain 26.80 acquired from the EPSAG Culture Collection of Algae. The strain was cultured in $500 \mathrm{~mL}$ Erlenmeyer flasks closed with semi-permeable lids. Cultures were maintained in COMBO medium. ${ }^{52}$ Every week, $30 \mathrm{~mL}$ of fluid was removed from the flasks and placed in new Erlenmeyer flasks in a 10:1 medium-algae ratio. Algal cultures were maintained in an 16L:8D photoperiod at $19^{\circ} \mathrm{C}$. Because we clonally propagated a single strain of Cryptomonas sp., there is no genetic variation among our treatments.

Daphnia magna was used as the predator species. Daphnia is a common predator of Cryptophyta. Daphnia was cultured in $600 \mathrm{~mL}$ beakers filled with COMBO medium at $19^{\circ} \mathrm{C}$. Medium was replenished twice a week. Simultaneously, $5 \mathrm{~mL}$ of a 2 to 3 week old Cryptomonas sp. culture was added as a food resource.

Crypotmonas sp. and Daphnia magna are very abundant in most waters of Western Europe. Daphnia pulex grazes on phytoplankton, algae and bacteria. The amount of studies investigating effects of predator cues of Daphnia is limited. However, it has been shown that the distribution of phytoplankton in naturally occurring freshwater varies in response to the resident zooplankton community. ${ }^{53}$ Moreover, Latta et al. ${ }^{54}$ found that predator cues induced phototactic movement of Chlamydomonas reinhardtii that resulted in a higher algae density at the surface than in untreated water. By remaining at the water surface, the algae could avoid its grazer since the grazers avoid the highest water layers in the presence of predators.

Another algae, H. akashiwo, was reported to have higher swimming speeds and vertical velocities in the presence of a ciliate predator. The direction was upward in the presence of a halocline. The ciliate was not able to persist at low salt concentrations and, as such, this area was a safe zone for the algae. In the absence of a halocline, the direction of the algae was downward, while the ciliates were aggregated at the top of the tank. ${ }^{55}$

\section{Experimental setup}

We constructed a two-patch system consisting of $21.5 \mathrm{~mL}$ Eppendorf tubes connected by a Fluoroelastomer tube (length $2.5 \mathrm{~cm}$, inner diameter $4 \mathrm{~mm}$, outer diameter $6 \mathrm{~mm}$ ). Fluoroelastomer is black and non-transparent and thus avoids light penetration in the connection tube. As a result, this part of the set-up is considered as the hostile matrix. 


\section{Treatments}

First, $2.78 \mathrm{~mL}$ of $\mathrm{a} \mathrm{PO}_{4}{ }^{3-}$-poor medium was added to all two-patch systems, independent of the treatment. After filling the tubes, the connection was closed. $140 \mu \mathrm{L}$ algae were added to each origin patch after manipulating resources and predator cues.

Resource availability The resource was a COMBO medium, ${ }^{52}$ with phosphate as limiting resource. For the standard resource treatment (standard RA), $11.2 \mu \mathrm{L}$ of a $\mathrm{KH}_{2} \mathrm{PO}_{4}{ }^{3-}$-solution was added to origin patches to obtain a $\mathrm{PO}_{4}{ }^{3-}$-rich medium. For the low resource treatment, $11.2 \mu \mathrm{L}$ Milli-Q water was added to origin patches to obtain a $\mathrm{PO}_{4}{ }^{3-}$-poor medium. Phosphate concentration was therefore: standard $\left[\mathrm{KH}_{2} \mathrm{PO}_{4}{ }^{3-}\right]=8.71 \mathrm{mg} \mathrm{L}^{-1}$, low $\left[\mathrm{KH}_{2} \mathrm{PO}_{4}{ }^{3-}\right]=0.871 \mathrm{mg} \mathrm{L}^{-1}$.

$11.2 \mu \mathrm{L}$ of a $\mathrm{KH}_{2} \mathrm{PO}_{4}{ }^{3-}$-solution and was added to all target patches to obtain a $\mathrm{PO}_{4}{ }^{3-}$-rich medium.

Predator cues Grazer kairomone water was created by isolating 40 individual Daphnia adults and placing them in $100 \mathrm{~mL}$ of filtered COMBO medium ${ }^{52}\left(\mathrm{PO}_{4}{ }^{3-}\right.$-limited: $\left.10 \%\right)$ for $18 \mathrm{~h}$. A similar procedure was followed by Latta et al. ${ }^{54}$ Prior to use the kairomone water was filtered through a 165 um nitex mesh to remove particulate matter and the Daphnia. For the predator cues treatment (yes PRED), 0.695 mL of medium was replaced with $0.695 \mathrm{~mL}$ of a predator cues $\mathrm{PO}_{4}{ }^{3-}$-poor medium in the origin patch before adding $\mathrm{KH}_{2} \mathrm{PO}_{4}{ }^{3-}$-solution and algae.

The experiment was run for $18 \mathrm{~h}$ (approx. 5\% dispersal in control treatment: standard RA, no PRED). The acclimation phase was $4.5 \mathrm{~h}$ (that is, $25 \%$ of the duration of the experiment). There were 6 replicates per treatment. The whole experiment was run in 2 blocks, each consisting of 3 replicates. The blocks differed in incubator and the moment the experiment was started $(1 \mathrm{~h})$.

\section{Data collection}

Both patches were sampled after the dispersal phase. $1.2 \mathrm{~mL}$ per patch was removed and stored in $1.5 \mathrm{~mL}$ Eppendorf tubes. To preserve the samples and fixate the algae, lugol was added. Densities were determined using a $1 \mathrm{~mL}$ counting chamber. Moreover, a sample of $8 \mu \mathrm{L}$ from the origin patch was taken for video analysis (concentrations were too low to take samples of the target patch for video analysis). For measuring dispersal, cell numbers were acquired using a counting chamber. 


\section{Grey field slug — Deroceras reticulatum}

Authors Maxime Dahirel, Armelle Ansart and Luc Madec

\section{Study organism and predator}

The grey field slug, Deroceras reticulatum (Müller; Gastropoda, family Agriolimacidae) is one of the most common and economically important crop pests of temperate regions, ${ }^{56}$ and is occasionally encountered in forests. ${ }^{57}$ Slugs used in this experiment were caught using non-baited traps (De Sangosseß), Pont-duCasse, France) in the spring of 2016 in a wheat field in Menetou-Salon, France $\left(47^{\circ} 16^{\prime} 1^{\prime \prime} \mathrm{N}, 2^{\circ} 22^{\prime} 23^{\prime \prime}\right.$ E). Slugs were housed in transparent polyethylene boxes $(26.5$ x 13.5 x $8.5 \mathrm{~cm}$, approx. 50 randomly assigned slugs per box) lined with synthetic foam kept saturated in water. Egg carton pieces saturated in water were added to be used as shelters. All rearing and experimental boxes were kept under controlled conditions $\left(10 \pm 1{ }^{\circ} \mathrm{C}, 12 \mathrm{~L}: 12 \mathrm{D} ;^{58}\right)$. Slugs were fed ad libitum with commercial snail food (cereal flour supplemented with calcium, Hélinoveß, Le Boupère, France), cucumber (Cucumis sativus Linnaeus) and lettuce (Lactuca sativa Linnaeus). Boxes were cleaned, and the shelters and lining changed, twice a week.

The parallel-sided ground beetle Abax parallelepipedus (Piller \& Mitterpacher; Coleoptera, family Carabidae) was used as our model predator. This large generalist predatory beetle is present in forests and agricultural landscapes in western Europe, although it is rarer in the latter. ${ }^{59,60}$ Large generalist carabid beetles such as A. parallelepipedus are predators of slugs, including Deroceras reticulatum. ${ }^{47,61,62}$ Slugs have both much smaller home ranges (in the range of $1 \mathrm{~m}^{2}$ versus 14 to $650 \mathrm{~m}^{2}$ depending on environments, ${ }^{63,64}$ ) and slower movement speeds than Abax parallelepipedus (maximal speed approx. $10 \mathrm{~cm} \mathrm{~min}^{-1}$ versus up to $20 \mathrm{~cm} \mathrm{~s}^{-1}$, M. Dahirel, personal observations on tested slugs, $\left.{ }^{65}\right)$. D. reticulatum has previously been shown to be able to detect and avoid several predatory ground beetle species based on various olfactory cues. ${ }^{51,58,66}$ Beetles were caught by hand in April 2016 in a small forest near Pléguien, France $\left(48^{\circ} 36^{\prime} \mathrm{N}, 2^{\circ} 56^{\prime} \mathrm{W}\right)$. They were then maintained in controlled conditions $\left(20^{\circ} \mathrm{C} \pm 1{ }^{\circ} \mathrm{C} ; 16 \mathrm{~L}\right.$ : 8D), in $5.5 \mathrm{~cm}$ high cylindrical boxes (diameter: $9 \mathrm{~cm}$ ) with $0.5 \mathrm{~cm}$ of moistened soil at the bottom (one to two beetles per box). Beetles were fed ad libitum with moistened dried cat food and apple slices; in addition, one live Deroceras reticulatum slug was provided per beetle once a week.

\section{Experimental setup}

Two-patch systems were built in $40 \times 13 \mathrm{~cm}$ transparent plastic boxes (height: $9 \mathrm{~cm}$ ). Boxes were divided in two $10 \times 13 \mathrm{~cm}$ patches and one central $20 \times 13 \mathrm{~cm}$ matrix, separated by plastified cardboard walls. Two $13 \times 1 \mathrm{~cm}$ slots were left open in each cardboard wall to allow slugs to leave and enter patches. One feeder (plastic bottle cap) and one shelter (wet egg carton piece) were present in all patches, in addition to $1 \mathrm{~cm}$ of humid soil at the bottom. The between-patch space was left empty and dry. In preliminary tests (ad libitum food, no predator cues; see below), this setup resulted in between 20 and 30\% of dispersers after 
3 days, which is roughly the time most slugs stopped dispersing away from release in a release-recapture study. ${ }^{63}$

\section{Treatments}

Ten randomly chosen slugs were released per two-patch system, in the origin patch ; this density (0.13 per $\mathrm{m}^{2}$ ) is at the low end of the wide range of naturally observed population densities, ${ }^{57,67}$ as higher densities are difficult to manage in controlled conditions. We only used slugs with fresh masses over $200 \mathrm{mg}$ prior to the experiments, to ensure all individuals used were sexually mature/ reaching sexual maturity. ${ }^{58}$ We tested 5 replicates per combination of predator cue presence (yes/no PRED) and food restriction (standard/low RA) treatments, so 20 replicates and 200 slugs overall (see below for details on the implementation of the treatments). Due to logistical constraints, replicates were tested in two sessions of ten (start dates: May 5 and 19, 2016); all four possible treatments were roughly equally distributed between these two sessions. Slugs were placed in the origin patch, which was then closed for $24 \mathrm{~h}$ using an upside-down box of the same size. Whatman papers, with or without predator cues depending on the treatment (see below), was placed at the same time in shelters $(7 \times 7 \mathrm{~cm})$ and around feeders $(1.5 \mathrm{~cm}$ wide strip) in the origin patch, so that slugs had to crawl on them on their way to eat or rest. Lettuce was also added to feeders based on treatment and slug body mass (see below). After these 24 hours of acclimation, the origin patch was opened and slugs left free to disperse for 3 days. Whatman papers were left in place, feeders in the origin patch were refilled with food for 3 days (actual quantity depended on treatment), and feeders in the target patch were filled with enough lettuce to sustain all slugs for 3 days.

Resource availability Food consumption of Deroceras reticulatum under optimal availability conditions was estimated by placing slug pairs of known fresh mass for $24 \mathrm{~h}$ in $5.5 \mathrm{~cm}$ high cylindric boxes (diameter: $9 \mathrm{~cm}$ ) with $16 \mathrm{~cm}^{2}$ of lettuce per box $(\mathrm{N}=10$ pairs of slugs). Slugs consumed on average 10.02 x slug mass $(\mathrm{g})+0.08 \mathrm{~cm}^{2}$ of lettuce per day per slug $\left(R^{2}=0.71\right)$. Slugs in the standard RA treatments were then provided with the quantity they were expected to consume during the experiment plus $24 \mathrm{~h}$ of surplus, based on the above linear equation. Slugs in the low RA treatments were provided with $10 \%$ of the expected quantity; preliminary observations showed that $-90 \%$ was the strongest restriction that did not result in outright cannibalism attempts, as opposed to mere agonistic interactions, within the duration of the experiment. Food consumption was measured as the difference between the lettuce leaf surface present at the end of the $24 \mathrm{~h}$-acclimation period and the surface present at the end of the 3-day dispersal test.

Predator cues Deroceras reticulatum, like most land gastropods, has poor vision and audition, and relies primarily on chemical cues to apprehend its environment. ${ }^{50}$ Predator cues isolation was inspired by. ${ }^{58}$ Ten $A$. parallelepipedus beetles were left to walk one night $(10 \mathrm{~h})$ in an otherwise empty $22 \mathrm{x} 17.5 \mathrm{x}$ 
$9.5 \mathrm{~cm}$ box lined with Whatman paper strips (total surface: $420 \mathrm{~cm}^{2}$ of paper per box). Pristine Whatman paper strips were used as controls. Before being used in our experiments, this cue isolation method was tested following. ${ }^{6}$ Briefly, $26.5 \times 13.5 \times 8.5 \mathrm{~cm}$ test boxes were divided in two darkened shelters with a small $1 \mathrm{~cm}$ entrance and one central lit part. Wet egg cartons pieces and a feeder with commercial snail food were present in both shelters. In each test box, one randomly chosen shelter had a $1.5 \mathrm{~cm}$ wide Whatman paper strip with predator cues placed at its entrance; the other had a control paper strip. Slugs were placed, one at a time, in the lit central part of their box and were left free to move for $24 \mathrm{~h}$. After this time, most slugs were found in the control shelter instead of the one with the predator cue $\left(90 \% ; 18\right.$ out of $\left.20 ; \chi^{2}=12.8 ; p<0.001\right)$. Four other carabid taxa were tested at the same time using the same protocol and sample size (Poecilus cupreus (Linnaeus); Nebria brevicollis (Fabricius); mixture of Pterostichus madidus (Fabricius) and P. melanarius (Illiger)); A. parallelepipedus was the one eliciting the strongest response, and the only one in which all individual beetles ate all provided live slugs within $24 \mathrm{~h}$.

\section{Data collection}

The number of dispersers (i.e., individuals in the target patch) and residents (individuals in the origin patch) in each tunnel was recorded once after 3 days. 


\section{Amphipods - Dikerogammarus villosus and Gammarus fossarum}

Authors Chelsea J. Little, Emanuel A. Fronhofer and Florian Altermatt

\section{Study organisms and predator}

We used two amphipod (Crustacea, Amphipoda) species as study organisms: Gammarus fossarum (Koch) and Dikerogammarus villosus (Sowinsky). Amphipods are key macroinvertebrates in stream, river, and lake food webs because of their dual roles in shredding terrestrial detritus and serving as food items for larger organisms. Amphipods show a range of responses to fish predation, including reduction of drifting behavior, drifting primarily at night-time, finding refuge in benthic macrophytes, remaining in sediments, or simply sitting motionless to avoid visual detection; by contrast, the presence of macroinvertebrate predators can increase drifting behavior. ${ }^{68}$ In general, habitat complexity provides a respite from fish predation. ${ }^{69}$ Amphipods have two main modes of dispersal: a more active swimming or crawling mode, where $25-33 \%$ of individuals disperse, ${ }^{70,71}$ and a less-active drifting mode of dispersal. Because drift is not relevant in our experimental setup since there is no current (see below), we focus on the more active form of dispersal.

G. fossarum is a small freshwater amphipod native and common to central Europe. In November 2016 we collected individuals by kicknet from the third-order Sagentobelbach stream in Dübendorf, Switzerland $\left(47.39^{\circ} \mathrm{N}, 8.59^{\circ} \mathrm{W}\right)$. We selected adults in medium and large size classes, and later distributed them into experimental units such that distributions of size classes was uniform across the experiment. It is impractical to identify individuals by sex in the field/while living, except by separating precopulatory pairs; perhaps partially due to the season, we found only a few such pairs, and thus collected individuals without regard to sex. We assume that allocation of individuals to treatments was relatively even across the experiment. Amphipods were brought to the lab and placed in large holding containers of approx. 500 individuals, where they were gradually brought up to $18^{\circ} \mathrm{C}$, fed alder (Alnus glutinosa (Gaertner)) leaves (which had been conditioned in stream water with natural microbial and fungal communities for six days) ad libitum, and maintained for two and a half days before being allocated to experimental units.

D. villosus is a larger freshwater amphipod native to the Ponto-Caspian region which has established itself through the Rhine catchment in the last two decades. ${ }^{72}$ In January 2017 we collected individuals by kicknet from Lake Constance at Kesswil, Switzerland $\left(47.60^{\circ} \mathrm{N}, 9.32^{\circ} \mathrm{W}\right)$. Collection with respect to size and sex, and maintenance in the laboratory before the experiment, was identical as for G. fossarum.

The European perch (Perca fluviatilis (Linnaeus)) was used as a predator for both amphipod species. Perch is a highly mobile predator in lakes, and feeds on both other fish and on macroinvertebrates. Gammarid amphipods can make up a large part of the diet of lake perch, ${ }^{73-75}$ and young adult perch can provide strong top-down control of macroinvertebrate biomass. ${ }^{69}$ In the lower Rhine, the diet of perch has shifted substantially with the arrival of non-native species, and includes more non-native amphipods. ${ }^{76}$ 
We obtained fresh-caught, dead perch (Fischerei Grieser, Obermeilen, Switzerland for experiments with G. fossarum and Braschler's Comestibles, Zurich, Switzerland for experiments with D. villosus) and immediately swabbed the sides of the fish with cotton balls to capture mucus and the chemical cues contained therein (see below for details).

\section{Experimental setup}

Two-patch systems were built using $3 \mathrm{~L}(198 \times 198 \mathrm{~mm})$ polypropelene boxes connected by approx. $30 \mathrm{~cm}$ of silicon tubing with an inside diameter of $20 \mathrm{~mm}$ (previous observations with multiple tubing sizes showed that this diameter led to $17 \%$ dispersal of $G$. fossarum from control treatments (i.e. standard RA, no PRED) over a five-hour period; Little and Fronhofer, unpublished data). All boxes contained either alder leaves or imitation cloth leaves (see below for details) as well as a plastic imitation macrophyte to provide shelter and habitat complexity. Origin and target patches were randomized with respect to directionality. All boxes/patches were covered with a black lid to reduce light permeability, while the connection tube/matrix was left uncovered. Tests showed that when large tanks were half-covered with the shading lid, only $20 \%$ of G. fossarum stayed in the unshaded portion, thus preferring a shaded habitat.

\section{Treatments}

At the beginning of an experiment, 20 amphipods were placed in each origin patch. We tested ten replicates per combination of predator cue presence (yes/no PRED) and food restriction (standard/low RA) treatments, so 40 tunnels and 800 amphipods per species (see below for details on the implementation of the treatments). Due to logistical constraints, experiments on the two species were run separately ( $G$. fossarum start date: 10 November, 2016; D. villosus start date: 26 January, 2017).

Twenty-four hours before amphipods were placed in the experimental units, real or imitation leaves were placed in the origin and target patches (see below). The connection tube was shut using clamps to prevent floating movement of resources between the patches. At the same time as amphipods were placed in the origin patches, cotton balls containing predator cues, or clean control cotton balls, were also placed in the origin patches (see below). After 30 minutes of acclimation, the connection tube was opened and amphipods were left free to disperse for $4.5 \mathrm{~h}$ (G. fossarum) or $7 \mathrm{~h}$ (D. villosus). At the end of the dispersal phase, the connection tubes between origin and target patches were closed again with clamps and all amphipods were removed from the patches.

Resource availability During dispersal trials, amphipods were fed the same alder leaves they were exposed to during maintenance. Origin patches in the standard RA treatment, and target patches in all treatments, were provided with $1.5 \mathrm{~g}$ dry weight of alder leaves. Because amphipods can survive for at least two weeks under starvation conditions, ${ }^{77}$ we decided to use a complete food restriction and 
offered only imitation cloth leaves in the restricted-food treatment. The number of imitation leaves were chose to roughly match the surface area provided by the alder leaves in the standard RA treatment, in order to minimize any confounding effects of amphipods using the leaves themselves as habitat or hiding spots. ${ }^{78}$ Food consumption was not measured in this experiment because previous experiments in the same laboratory conditions showed that $G$. fossarum consume $0.35 \mathrm{mg}$ dry weight of alder leaves per mg dry weight of amphipod per day, and D. villosus $0.5 \mathrm{mg}$ dry weight of alder leaves per mg dry weight of amphipod per day; with only a few hours of experimental duration, it would be difficult to measure leaf consumption in the experimental units with sufficient precision to detect differences between treatments, if they did exist. $1.5 \mathrm{~g}$ dry weight of alder leaves were provided in all target patches.

Predator cues Cotton balls wiped on the sides of freshly killed, commercially caught P. fluviatilis to collect their mucus and the contained chemicals were used as predator cues. Cotton balls were frozen at $-20^{\circ} \mathrm{C}$ until the day of the experiment. Conspecific amphipods have been shown to alter their activity in the presence of fish cues, ${ }^{79-81}$ and a pilot experiment showed that these perch predator cues reduced dispersal rates from $5 \%$ to $0 \%$ from non-food-limited patches by $G$. fossarum. One cotton ball was placed in each origin patch in the yes PRED treatment patches, and a clean, sterile cotton ball (which had also been frozen at $-20^{\circ} \mathrm{C}$ ) was placed in the origin patch in no PRED treatment patches.

\section{Data collection}

The number of dispersers (i.e., individuals in the target patch) and residents (individuals in the origin patch) in all experimental units was recorded once after the dispersal period was concluded. 


\section{Paramecium caudatum}

Authors Florent Manzi and Oliver Kaltz

\section{Study organism and predator}

We used Paramecium caudatum as focal species (prey) and Didinium nasutum as predator. The Paramecium were taken from a long-term selection experiment on dispersal (O. Kaltz, unpubl. data; see below); the founder population for this experiment comprised a mix of 20 clones, but at the time of the present experiment, individual selection lines most likely consisted of single clones. Preliminary analysis indicates that different clones are fixed in high-dispersal and low-dispersal selection lines. Didinium was obtained from Sciento (strain P220).

This predator-prey system naturally occurs in freshwater environments, with Didinium feeding on different species of the genus Paramecium. ${ }^{82}$ Paramecium aurelia shows predator-induced dispersal in the presence of a flatworm predator. ${ }^{83}$ Other ciliate species can detect the presence of predators by direct membrane contact ${ }^{84}$ or use the hydrodynamic disturbance induced by cilia motion. ${ }^{85}$ In a pilot experiment, we found a tendency of increased dispersal of our P. caudatum strains exposed to a filtrate prepared from a Didinium culture (F. Manzi, unpubl. data). This suggested a plastic dispersal response mediated by chemical cues.

Both Paramecium and Didinium cultures were maintained in temperature-controlled incubators, at $23{ }^{\circ} \mathrm{C}$ in the dark. For the Paramecium cultures, we used an organic lettuce medium $(1 \mathrm{~g}$ of dried lettuce suspended in $1.5 \mathrm{~L}$ of Volvic ${ }^{\mathrm{TM}}$ mineral water), supplemented with the bacterium Serratia marcescens. ${ }^{86}$ The Didinium were regularly (2-3 times per week) fed with a mix of Paramecium from the abovementioned selection experiment.

Similar to Fronhofer \& Altermatt ${ }^{87}$ the long-term lines of Paramecium were going through alternating cycles of dispersal $(3 \mathrm{~h})$ and logistic growth $(7 \mathrm{~d})$, for approximately 1 year. For the present experiment, Paramecium were taken at the end of a growth cycle, when populations had reached carrying capacity. In high-dispersal selection treatments, non-dispersing Paramecium are discarded, and only dispersing Paramecium are retained, propagated for one week and then subjected to a new round of $3 \mathrm{~h}$ dispersal, and so on for 1 year. In the low-dispersal treatment, only the non-dispersing Paramecium are retained and used for a new growth/dispersal cycle. The experiment was conducted at $23^{\circ} \mathrm{C}$. We used independent Paramecium selection lines from each of two long-term directional selection treatments: high-dispersal lines and low-dispersal lines.

\section{Experimental setup}

The two-patch systems consisted of $13 \mathrm{~mL}$ plastic tubes with a round bottom, connected by silicone tubing (length: $5 \mathrm{~cm}$, diameter: $0.8 \mathrm{~mm}$ ), allowing dispersal of Paramecium between the two patches. 
The connection can be opened and blocked by means of plastic clamps.

To measure dispersal, Paramecium were filled in one of the two tubes (origin patch), while the connection between the two tubes was blocked. After an acclimation period of 30 min (approx. 17\% of the duration of the dispersal phase), the clamps were removed for three hours, during which time the Paramecium could freely swim between tubes. Then the clamps were put back to block the connection.

The target patch always contained $13 \mathrm{~mL}$ of standard medium. The composition of the origin patch varied according to treatment (see below). The connection between the tubes (i.e., the matrix) was filled with sterile mineral water $\left(\right.$ Volvic $\left.^{\mathrm{TM}}\right)$. This was achieved by first filling the entire two-patch system with water, then isolating the matrix from the patches by way of clamping, and replacing the water in the patches by medium and Paramecium, according to treatments.

\section{Treatments}

We used a total of 12 Paramecium selection lines of (6 lines from the 'high-dispersal' treatment and 6 lines from the 'low-dispersal' treatment) for the experiment. Each line was used at its 'natural' density (i.e., density reached after one week of culture) and we did not correct the total number of individuals present in the origin patch of a given replicate.

Prior to the experiment, two $25 \mathrm{~mL}$ samples from each selection line were centrifuged for $30 \mathrm{~min}$ at $1500 \mathrm{~g}$, and then approx. $22.5 \mathrm{~mL}$ of the supernatant discarded. For one sample, the concentrated Paramecium were then resuspended in standard medium to give a new total volume of $15 \mathrm{~mL}$; from this volume the two high-resource replicates were established in the experiment (see below). The other sample was resuspended in sterile mineral water to give two low-resource replicates.

On average, the final number of individuals introduced in the patch or origin was $1093 \pm 96$ s.e. There was no significant difference between high-dispersal and low-dispersal lines $(p>0.2)$.

We ran a total of 12 independent replicates for each treatment, giving a total of 48 replicates, with each selection line represented by one replicate in each treatment (2 RA treatments x 2 PRED treatments $\mathrm{x} 2$ selection origins $\mathrm{x} 6$ selection lines). The experiment was organised in six blocks of 8 replicates, with each treatment replicated twice per block. There was a delay of approx. 35 min between the start of each block. The entire experiment was conducted within one day.

Resource availability The resource consumed by Paramecium is the actively hunted bacterium Serratia marcescens. This bacterium is motile and may be able to disperse between patches. We did not establish whether this actually happened during the experiment.

To set up standard and low RA treatments, we centrifuged our Paramecium cultures (see above) and discarded almost all the supernatant, thereby removing (unsedimented) Serratia. In the standard RA treatment, the origin patch contains $6 \mathrm{~mL}$ of Paramecium (previously centrifuged, retrieved from 
the pellet and diluted in fresh culture medium) and $3.5 \mathrm{~mL}$ of either predator filtrate or control filtrate (see below). The volume is completed to $13 \mathrm{~mL}$ using $3.5 \mathrm{~mL}$ of 'high food' medium. High food medium consisted of sterilised lettuce medium (90\% volume), to which Serratia from a stock culture was added as a food resource (10\% volume). Prior to use, the medium was filtered through two layers of sterile medical gauze to remove larger lettuce particles.

In the low RA treatment, the origin patch contains $6 \mathrm{~mL}$ of Paramecium (previously centrifuged, retrieved from the pellet and diluted in sterilized water) and $3.5 \mathrm{~mL}$ of either predator filtrate or control filtrate. The volume is completed to $13 \mathrm{~mL}$ using $3.5 \mathrm{~mL}$ of sterilized water.

We did not quantify the density of Serratia in our resource treatments. Due to our dilution protocol, the Serratia content in the low RA treatment was reduced by c. $70 \%$ relative to the standard RA treatment (which corresponds to ad libitum conditions). In a growth assay prior to the main experiment, the low RA treatment reduced Paramecium population growth rate by $80 \%$ (data not shown).

Predator cues Other ciliate species (e.g., Euplotes octocarinatus) detect the presence of predator by direct membrane contact or use the hydrodynamic disturbances induced by cilia motion. ${ }^{84,85}$ Here, we make the assumption that $P$. caudatum detect the presence of Didinium through the use of chemical cues.

We combined $10 \mathrm{~mL}$ from each of 10 populations containing both Paramecium and Didinium at variable densities (overall, c. 5 x 103 Paramecium and Didinium, respectively). These cultures had been set up several weeks prior to the experiment, and were regularly supplied by a mix of Paramecium from high- and low-dispersal selection lines to maintain the Didinium populations. The combined populations were centrifuged at $1500 \mathrm{~g}$ for $30 \mathrm{~min}$. The supernatant was retrieved and filtered through a micropore filter $(0.2 \mu \mathrm{m})$. This filtrate served as Didinium cue ('predator filtrate'; yes PRED).

In the same way, we prepared the control filtrate (no PRED). Here, our lettuce culture medium served as a basis, without any addition of Serratia, Paramecium or Didinium. Prior tests had shown that filtrates from pure medium and filtrates from Paramecium cultures (without Didinium) did not significantly differ in the effect on dispersal; and the effect of both types of filtrate were not significantly different from a sterile water control (results not shown).

A pilot experiment (data not shown) indicated a positive relationship between dispersal rate and filtrate concentration $(0 \%-10 \%-100 \%)$, as well as the number of Didinium individuals added $(0-5-$ $25)$.

\section{Dispersal and data collection}

Paramecium were allowed to disperse for $3 \mathrm{~h}$, until the connection between origin and target patches was blocked. We then took $300 \mu \mathrm{L}$ samples from the patch of origin and $1 \mathrm{~mL}$ samples from the target patch. 
The number of individuals in these samples was counted under a dissection microscope (20x). From these measurement we extrapolated the total number of individuals in both tubes in a total of $13 \mathrm{~mL}$. Dispersers are defined as individuals that are present in the target patch at the end of the $3 \mathrm{~h}$ period. Replication during the dispersal period can be neglected. ${ }^{88}$ 


\section{European minnow — Phoxinus phoxinus}

Author Simon Blanchet

License E-2016-130

\section{Study organism and predator}

The European minnow (Phoxinus phoxinus) is a small bodied-size (max. length of approx. $8 \mathrm{~cm}$ ) freshwater fish species widespread in Europe. Its main predators include predatory fish as well as piscivorous birds and mammals. We here used 192 adults (3-years, approx. $6.2 \mathrm{~cm}$ long, min: $4.5 \mathrm{~cm}$ max: $8.4 \mathrm{~cm}$ ) fish originating from a single population raised in artificial lakes in Brittany (France). Minnows were reared for two weeks in a $1100 \mathrm{~L}$ external tank and feed ad libitum with pellets and frozen bloodworms.

We used chemical cues from a common predatory fish (the Eurasian perch, Perca fluviatilis) that is widely co-occurring with minnows throughout their ranges. A single perch (approx. $20 \mathrm{~cm}$ in length) was captured and maintained alive in a $800 \mathrm{~L}$ external tank.

We estimated minnow and perch home ranges from stationary distance obtained from the 'fishmove' $\mathrm{R}$ package $\mathrm{e}^{89}$ and that we considered as the home range radius. We estimated this stationary distance for 30 days using a species and size specific approach for the perch (length $=200 \mathrm{~mm}$ ) and a size specific approach for minnows (length $=80 \mathrm{~mm}$ ), because species information was unavailable. Minnows have a smaller home range than the perch $\left(80 \mathrm{~m}^{2}\right.$ for minnows, $5808 \mathrm{~m}^{2}$ for Eurasian perch). The mobility, estimated as sprint speed, was also smaller for minnows than for the perch (minnow: approx. $40 \mathrm{~cm} \mathrm{~s}^{-1}$, estimated on close species from; ${ }^{90} 183 \mathrm{~cm} \mathrm{~s}^{-1}$ for other perch species, $\left.{ }^{91}\right)$. On top of dispersal, minnows use schooling as an efficient antipredator defence. ${ }^{92}$

\section{Experimental setup}

The experimental setup consisted of three-patch systems made of three circular $1100 \mathrm{~L}$ plastic tanks connected with opaque pipes of $16 \mathrm{~cm}$ in diameter and $120 \mathrm{~cm}$ in length. Each of the three tanks was filled with tap water at least 4 days before the experiment started. The bottom of each tank was left empty in all tanks. At the end of the last pipe, we added a cut plastic bottle to create an anti-reverse device and prevent dispersers to return in origin patches.

\section{Treatments}

We created 24 populations made of 8 fish that were from the same cohort and from a single strain to avoid age and strain effects. Individuals were not sexed before or after the experiment. We used a $2 \times 2$ factorial design, crossing resource availability (RA) and predation risk (PRED) with two levels each. We ran the eight replicates in 2 blocks (November 3rd to 10th 2016 and December 2nd to 9th 2016). Three 
days before the experiment started, groups of eight minnows were encaged in $5 \mathrm{~L}$ bottles directly emerged in their rearing tank. The fish were then acclimatized for 24 hours with RA and PRED treatments in the origin patch before opening the corridors. After 24 hours, connections were opened and we monitored dispersal movements as described below.

Resource availability RA includes two treatments a low and standard RA treatment. The resources are manipulated through the addition of frozen bloodworms (approx. 8g) in the standard RA treatment whereas we did not introduce food in the low RA treatment. It allows creating a large difference in food availability between treatments.

Predator cues Minnows have already been shown to behaviourally react to the odour of their natural predators, ${ }^{93}$ although these reactions were exacerbated when predatory odours were mixed with Schreckstoff $\left(;^{94}\right.$ Blanchet S., unpublished data). To maximize the potential reaction of minnows, we therefore mixed these two types of alarm cues in our experiment. Specifically, for each tank with predator cues, $200 \mathrm{~mL}$ of water was extracted from the perch tank and mixed (using a grinder) with the skins of two freshly dead minnows. ${ }^{94}$ We used this solution to mimic the presence of a dangerous predator in our experiment.

\section{Dispersal and data collection}

We allowed fish to disperse for 7 days during the first block and 10 days during the second block. The difference of time was statistically controlled through a block random intercept. All tanks were then emptied out to count the number of fish in each tank. We considered the residents as the fish having been caught in the origin patch, while dispersers were those in the farthest tank from origin patch. It resulted into 125 fish used in the analyses. 


\section{Large white butterfly - Pieris brassicae, White-legged damselfly — Platyc- nemis pennipes}

Authors Delphine Legrand, Lieven Therry, Alexandre Vong, Staffan Jacob and Julien Cote

\section{License $\quad 09-2016-02$}

\section{Study organism and predator}

Pieris brassicae The large white butterfly (Pieris brassicae) is a widespread butterfly across Europe $^{95}$ We used 88 butterflies issued from a breeding population (2 generations of breeding initiated in the Metatron ${ }^{1}$ from clutches collected in 2016 in Ariège, France). Individuals were all measured for several phenotypic traits (see below), sexed and marked with a specific number on their wings.

The common toad (Bufo bufo) was used as our model predator. The species inhabits wet locations in Europe and is a common predator of many insects including adult butterflies. ${ }^{3}$ Eight toads were caught by hand near the Metatron and were maintained as described below.

We estimated that butterflies have a larger home range than the common toad $\left(8000-28000 \mathrm{~m}^{2}\right.$ for common toads, ${ }^{7}>300000 \mathrm{~m}^{2}$ for butterflies ${ }^{96,97}$ ). Mobility, estimated as flight speed for butterflies and sprint speed for toads, was higher for butterflies than for toads (butterflies: approx. $5 \mathrm{~ms}^{-1}$ for Pieridae species in natural conditions, ${ }^{98}$ and approx. $3 \mathrm{~ms}^{-1}$ for $P$. brassicae in experimental conditions, S. Ducatez pers. comm.; toad: approx. $\left.0.3 \mathrm{~ms}^{-1},{ }^{10}\right)$.

Platycnemis pennipes The white-legged damselfly (Platycnemis pennipes) is a widespread damselfly occurring from the Atlantic to the Jenisei river in Siberia and typically breeds in a wide range of aquatic habitats such as rivers, canals and fish ponds. ${ }^{99}$ We caught 160 damselflies near the Metatron ${ }^{1}$ in Ariège (France) in mid-July 2016. Individuals were all measured for several phenotypic traits, sexed and marked with a unique number on their wings. After marking all of them, individuals were transferred to 8 seminatural enclosures randomly, except for sexes which were distributed to obtain similar sex-ratio among enclosures. The common frog (Rana temporaria) was used as our model predator. The species inhabits wet locations in Europe and is a common predator of adult damselflies. ${ }^{100}$ Eight frogs were caught by hand in the same location as damselflies and were maintained as described below.

We estimated that damselflies have a larger home range than the common frog $\left(12500 \mathrm{~m}^{2}\right.$ for damselflies, ${ }^{101} 330-1500 \mathrm{~m}^{2}$ for common frogs $\left.{ }^{102,103}\right)$. Mobility, estimated as maximal flight speed for damselflies and sprint speed for frogs, was higher for damselflies than for frogs (frogs: $20-80 \mathrm{~cm} \mathrm{~s}^{-1}$, for another Rana species, ${ }^{104}$ damselflies: $140 \mathrm{cms}^{-1},{ }^{105}$ ). On top of dispersal, damselflies (including Platycnemis sp.) exhibit group oviposition to reduce predation risk at the oviposition site where frogs are encountered. ${ }^{106,107}$ 


\section{Experimental setup}

We used the Metatron, an experimental platform dedicated to the study of dispersal in terrestrial organisms, ${ }^{1}$ especially in flying insects. ${ }^{108}$ For each species separately, we used 16 enclosures of the Metatron (each $200 \mathrm{~m}^{3}, 10 \times 10 \times 2 \mathrm{~m}$ and covered with insect-proof nets) to create eight two-patch systems (i.e. two connected enclosures). We connected each origin patch (in which butterflies, respectively damselflies, were released) to a target patch using a corridor. Thus, butterflies and damselflies could either remain in the origin patch or freely cross a corridor to immigrate into the target patch, with the possibility of returns to the origin patch. However, the narrow, S-shaped $19 \mathrm{~m}$ long corridors were particularly challenging to cross (i.e., dark and warm conditions, low vegetation) and allow discrimination between disperser and resident individuals. ${ }^{1}$ We maintained the vegetation of higher height and added a small fenced water pond ( $25 \mathrm{~L}$ plastic patch, $60 \times 39 \times 16 \mathrm{~cm}$ ) in all origin and target patches. We have previously shown that these conditions allowed the discrimination of dispersal events in P. brassicae. ${ }^{108,109}$

\section{Treatments}

We created sixteen populations for each species made of 11 butterflies ( $55 \%$ females) or 20 damselflies (approx. $40 \%$ females) in each origin patch which represents a density at the lower range of naturally observed populations (butterfly: 5 - 800 individuals per $100 \mathrm{~m}^{2},{ }^{110}$ damselfly: 0.2 individual per $\mathrm{m}^{2}{ }^{111}$ ). After releasing individuals, we applied the food resources and the predator cues treatments. We used a 2 x 2 factorial design, crossing resource availability (RA) and predation risk (PRED) with two levels each, resulting into 2 replicates per species of each combination of treatments, tested in 1 block. The RA and PRED treatment were applied at the beginning of the acclimation phase. During the acclimation phase, which lasted 24 hours, corridors were kept closed to prevent any movements between origin and target patches. After 24 hours, corridors were opened and we monitored dispersal as described below.

Resource availability RA includes two treatments: a low and standard RA treatment with a large difference in food availability between treatments. For butterflies, we maintained the vegetation of low height in all origin and target patches and, in the standard RA origin patches only, we added 2 feeding flowerpots placed in the same corner, and host plant (fresh cabbages) in the centre of all patches. For damselflies, we kept enclosures in the low RA treatment with their natural insect community. In the standard RA treatment, we added approx. 100 fruit flies (mix of Drosophila species with body sizes comprised between 2 and $6 \mathrm{~mm}$ ) per enclosures and a fruit mixture with approx. 200 pupae emerging gradually throughout the experiment. Small Dipterans are the main prey for adult damselflies ${ }^{112}$ and fruit flies were already used as experimental food resources. ${ }^{113}$ We estimated the relative amount of flying insects after our experiment using a swiping net. The addition of Drosophila in the standard RA treatment resulted in an increased amount of $3-6 \mathrm{~mm}$ flying insects $\left(F_{1,6}=5.33, P=0.06, R^{2}=0.47\right)$, 
but not of flying insects smaller than $3 \mathrm{~mm}\left(F_{1,6}=0.02, P=0.90\right)$. As we did not find any fruit flies in the samples, the effect on $3-6 \mathrm{~mm}$ flying insects is likely an indirect effect of damselflies eating preferentially fruit flies rather than other flying insects.

Predator cues PRED includes a treatment, where two toads for butterflies or two frogs for damselflies were added in the fenced pond (yes PRED), and a treatment where the fenced pond was left empty (no PRED). The addition of toads or frogs produced both a visual and olfactory cue with no actual predation. Damselflies are known to select oviposition sites depending on the presence of predators ${ }^{114}$ and our predator treatment mimics this. Enclosures naturally contain several spiders, which are potential predators of damselfly. However, we removed most of them before the experiment and we also estimated their relative abundance after the experiment. We checked that adding this relative abundance in our statistical analyses did not change our results. As the literature is less abundant about predator cues in butterflies, we also added two individuals that were crushed in a tube in the yes PRED treatment for butterflies as alarm cues of predation, and an empty tube in no predation treatments.

\section{Dispersal and data collection}

After the opening of corridors, we monitored dispersal daily for 4 and 5 days for butterflies and damselflies respectively. To do so, two people entered and walked quietly for $5 \mathrm{~min}$ in each cage. When a butterfly or a damselfly was located, the observer recorded the individual number. This procedure is commonly used on these species ${ }^{1,108,109}$ and we did not observe any individuals leaving the enclosure during dispersal monitoring. We attributed a disperser status to individuals which moved at least once between patches and a resident status to individuals which never left the origin patch. We retained individuals which were alive after three days and excluded individuals which were never observed as their dispersal status is unknown. 


\section{Tetrahymena sp.}

Authors Staffan Jacob, Estelle Laurent and Nicolas Schtickzelle

\section{Study organism and predator}

We used two species of Tetrahymena ciliates (T. thermophila and T. elliotti), that is, freshwater approx. $50 \mathrm{~mm}$ long protists that actively swim and disperse using ciliae beating movements, foraging on bacteria in nature (N-America). They were cultured in the laboratory under standardized conditions: $23^{\circ} \mathrm{C}$ temperature, in a homogeneous nutrient broth (PPYE medium: 2\% Proteose peptone and 0.2\% yeast extract diluted in ultrapure water) and under strict axenic conditions (sterilized material and culture medium; all manipulations under a flow hood). Tetrahymena thermophila has been used as a model species to study different aspects of dispersal: e.g. intraspecific variation, ${ }^{115}$ dispersal syndromes and link with cooperation strategies ${ }^{116-119}$ phenotypic plasticity and density dependence, ${ }^{115,119}$ non-random and informed dispersal. ${ }^{119-121}$ The predator used was Blepharisma sp., a mobile predator that feeds on Tetrahymena ciliates.

\section{Experimental setup}

The experiments were conducted in two-patch systems consisting of two habitat patches $(1.5 \mathrm{~mL}$ standard microtubes), connected by a corridor ( $4 \mathrm{~mL}$ internal diameter silicon tube, $2.5 \mathrm{~cm}$ long) and filled with growth media. ${ }^{115-119,122}$ Cells were placed in the origin patch and the corridor was opened to allow dispersal towards the target patch. At the end of the dispersal time, the corridor was clamped to separate residents (cells remaining in the origin patch) from dispersers (cells that moved to the target patch).

For each Tetrahymena species, we used four isolated genotypes (T. thermophila strains: 18282-1, 18282-4, 18296-4, 19876-1; T. elliotti strains: 18470-2, 18765-1, 19484-1, 18662-4; Tetrahymena Stock Center, Cornell University, New York) that we mixed at equal density to obtain genetically variable populations (final cell density: 40000 cells $\mathrm{mL}^{-1}$ ). Genotypes were mixed just before the beginning of the experiment, and $200 \mu \mathrm{L}$ of each mix were inoculated in the origin patch of each two-patch system. After one-hour acclimation, corridors were opened to allow cells to disperse for four hours.

\section{Treatments}

Predator cues (yes/no PRED) and resource availability (standard/low RA) were manipulated in the origin patches, with 5 replicates per species and treatment.

Resource availability The RA treatment consisted in filling the origin patch with either standard resource concentration (standard RA) or $10 \%$ of standard concentration (low RA). The target patch 
was always filled with standard resource concentration. Furthermore, corridors were filled with water to create an unsuitable matrix containing no resource.

Predator cues The predator cues were obtained by filtering Blepharisma sp. culture at carrying capacity (i.e., 2 weeks old culture) using $0.2 \mu \mathrm{m}$ filters. $100 \mu \mathrm{L}$ of filtered predator culture, thus containing only chemicals from the predator culture, were then added in the origin patch of the two-patch systems for the yes PRED treatment. Since predators (Blepharisma sp.) were cultured on protozoan pellet $\left(0.46 \mathrm{~g} \mathrm{~L}^{-1}\right.$; Carolina Biological Supply) with bacteria (Serratia fonticola, Bacillus subtilis and Brevibacillus brevis), we performed preliminary analyses using a filtrate from a bacteria culture on protozoan pellet instead of predator cues, and found no effect on dispersal rate compared to the absence of cues.

\section{Data collection}

We used a standardized procedure to measure cell density and morphology based on automatic analysis of digital images. ${ }^{123}$ For each culture sample (i.e., a specific patch of a two-patch system), we pipetted five $10 \mu \mathrm{L}$ samples into a multichambered counting slide (Kima precision cell 301890), and took one digital picture of each chamber under a dark-field microscope. ${ }^{115-120,122}$ Digital pictures were analysed using ImageJ software (version 1.47, National Institutes of Health, USA, http://imagej.nih.gov/ij) to obtain the overall number of cells on the picture, from which the abundance in each culture tube was recomputed. ${ }^{123}$ 


\section{Two-spotted spider mite — Tetranychus urticae}

Authors Stefan Masier and Dries Bonte

\section{Study organism and predator}

The model species used for this experiment was the haplodiploid spider mite Tetranychus urticae Koch (Acarina: Tetranychidae), also known as two-spotted spider mite, belonging to the 'LS-VL' strain. The original population of T. urticae was collected in October 2000 from a botanical garden in Ghent (Belgium) where pesticides had not been used for at least ten years. ${ }^{124}$

Mites are phytophagous, aboveground generalist herbivores feeding on plant cell fluids. They are a common pest in gardens, fields and greenhouses and can feed on a large variety of plants species. The bean is well-known from the literature as one of the species the spider mites can feed on most efficiently, as it does not produce harmful chemicals when attacked (opposed to other plants like peppers or tomatoes). The average body length for an adult female is approx. $0.4 \mathrm{~mm} .^{125}$

Phytoseiulus persimilis Athias-Henriot is well-known as a voracious predator of T. urticae and can have a strong impact on the population dynamics of its prey. ${ }^{126,127}$ The close ecological relation between the two species is widely described, ${ }^{128}$ and this predator is also commonly used as a pest control in greenhouses. For these reasons, we were reasonably sure we could expect plastic response in the prey behaviour when faced with predator cues. ${ }^{129}$ Avoidance had been the most studied response, even if other reactions have been recently found in closely related species. ${ }^{130}$ We bought a vial with approx. 200 individuals from a professional seller and we raised them on bean plants infested by T. urticae for 1 generation before performing the experiment.

The two-spotted spider mites were raised on an optimal host, the common bean (P. vulgaris L., variety 'Prelude'). The plants were grown in an herbivore-free and pesticide-free walk-in climate room at $25^{\circ} \mathrm{C}$ and under a $16 / 8 \mathrm{~h}(\mathrm{~L} / \mathrm{D})$ photoperiod. Regularly, two-weeks old bean plants were moved to a second climatically controlled room $\left(25 \pm 1^{\circ} \mathrm{C}\right)$ with the same light regime and humidity where the population of mites was kept in open plastic boxes. The same conditions of light and temperature had been maintained for the whole duration of the experiment, except when explicitly noted.

Before the experimental setup, a set of adult females from the stock population of T. urticae was synchronized to avoid any possible age-related effect. In order to do so, 50 females had been randomly collected and placed on a $7 \times 7 \mathrm{~cm}$ square patch freshly cut from a bean leaf. After 48 hours, the females were removed and the leaf, mounted on a bed of moisturized cotton to keep it fresh, was placed into a heated cabinet to prevent contamination and provide optimal conditions for the development of the juveniles. The eggs were monitored daily until they reached the adult stage (approx. 7 days at $30^{\circ} \mathrm{C}$ ). We chose to select for the experiment only mated, one-to-two days old females of T. urticae, as they are well-known from the literature as the main dispersing stage in the mites life cycle. ${ }^{131,132}$ 


\section{Experimental setup}

To test for dispersal propensity, two-patch systems were composed as following. A 2.5 x $1.5 \mathrm{~cm}$ leaf square cut from a two-weeks-old plant of $P$. vulgaris have been mounted on a cotton bed and fixed in place using paper stripes. The cotton and the paper are constantly kept moist to avoid dehydration of the leaf and to prevent the mites from escaping. The paper strips also provided the additional value to stop the mites from crawling under the leaf, where they would have been at risk of drowning, and to protect the exposed edges of the leaf from dehydration. The origin patch was then connected to an identical target patch using a Parafilm® bridge, that constitutes an unsuitable environment for the spider mites. The length of the bridge was $8 \mathrm{~cm}$, as during pilot experiments this distance was shown to set a dispersal rate between $15 \%$ and $20 \%$.

\section{Treatments}

The population on the origin patch was composed by one-to-two days old adult, fertilized female spider mites, to maximize the number of individuals suitable for dispersal and subsequent analysis. All the individuals used for the experiment belong to the LS-VL strain. On the origin patch, 50 individuals were placed after applying treatments (see below), for a density of approx. 13 mites $\mathrm{cm}^{-2}$. This mimics a mild-to-severe infestation in natural conditions, and can stimulate the dispersion of individuals. ${ }^{133}$

We let the mites settle for 24 hours before connecting the bridges, and then left the spider mites free to disperse for the following 48 hours. We ran five different replicates of each combination of treatments at once. The replicates are independent one from the other as each time new individuals belonging to the synchronized offspring of stock females were used.

Resource availability From previous experiments, we assessed that a $2.5 \times 1.5 \mathrm{~cm}$ leaf square cut from a fresh bean leaf is an abundant source of food that can sustain approx. 50 mites for at least 48 hours. As such, it was used for the standard RA treatment. The leaves from two-week-old bean plants were cut from the plant, shaped accordingly and left on a wet layer of cotton for 48 hours to ensure hydration before the start of the experiment.

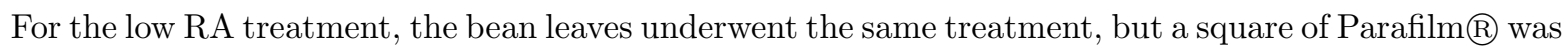
placed between the cotton bed and the leaf to isolate the leaf itself from the water. Doing so, the leaves withered and lost wet weight, presenting less food available for the animals to eat, as mites are not able to feed on plant tissues with a reduced percentage of water. The cotton had to be kept wet in the predator treatments (see below) to prevent individuals from escaping, so water was added to all the preparations to maintain the humidity levels constant through all the replicates and avoid adding an unwanted difference between the treatments. Pilot choice tests were performed in advance showing that, while mites do not significantly discriminate between fresh and 24-hours-old withered leaves, they show a marked avoidance 
for leaves that were left to wither for more than 48 hours.

The amount of food consumed by mites can be measured by counting the feeding scars left on the leaves and comparing the scarred area with the patch total area. ${ }^{134}$ This procedure had been largely applied in previous studies and it can be standardized using specific software tools. Before and after the experiment, a picture of every patch was taken and the pairs were digitally compared to evaluate the consumption rate and the per capita amount of consumed resource.

Predator cues Mites are known to be able to detect the presence of a predator mostly using chemical cues. In particular, hormones secreted by the predators seem to be the major cue for the spider mites to avoid leaves infested by predators. ${ }^{135,136}$ The origin patches with predator cues (yes PRED) were prepared by letting a sample of 5 predatory mites wander freely on the leaf cut for 24 hours right before the beginning of the experiment. This number was chosen to ensure abundant cues of the presence of $P$. persimilis were left on the leaf: it was shown that two-spotted spider mites are able to spot this amount of predators when present on a leaf and modify their behaviour accordingly. ${ }^{135}$ The predators were well-fed before being moved to the experimental patch, as no prey was present on the leaf to avoid confounding effects due to individuals feeding on the leaf, and thus potentially changing the starting condition of the patch, or leaving information on the previous population composition, eventually including relatedness. All alive predator mites and eggs were then removed before placing the experimental population, leaving only the webs and the fecal material left during their stay. Only patches presenting at least three alive predators and/or one egg were chosen for the experiment, to ensure that predators spent a significant amount of time on the leaf. For the removal, a thin paint brush was used; special attention was paid to ensure no contamination between the patches exposed to predators and the control ones by carefully cleaning the hairbrush with water and ethanol after each patch preparation.

\section{Data collection}

After dispersal time is over, the bridges were removed and the individuals crawling on origin and target patches and the bridge itself were visually counted. Individuals on the bridge were excluded from the analyses. A partial count was also performed roughly every 24 hours to ensure some intermediate data as well. 


\section{Common lizard - Zootoca vivipara}

Authors Laurane Winandy, Félix Pellerin, Lucie di Gesu, Delphine Legrand and Julien Cote

\section{License 2012-10 DREAL}

\section{Study organism and predator}

The common lizard (Zootoca vivipara; Jacquin 1787) is a small lacertid (adult snout-vent length: males, 40-60 mm; females, 45-75 mm) generally found in humid habitats throughout Eurasia. In this experiment, we used 112 two-year old lizards born in the lab (Ariège, France) and raised in $1100 \mathrm{~L}$ cattle tanks (diameter: $1.70 \mathrm{~m}$ ). Home tanks contained $20 \mathrm{~cm}$ of soil litter, dense vegetation, ten $50 \mathrm{~mL}$ Falcon tube in the litter and 3 half flower pots used as refuges, two small dishes for water and a regular addition of crickets. These conditions were highly suitable for lizards, as shown in previous experiments. ${ }^{34,137,138}$ As lizards spent their entire life in cattle tanks, they have never been exposed to real predators. Individuals were all marked at birth, ${ }^{137}$ sexed and transferred to our experiment dispersal two-patch systems (see below).

The green whip snake (Hierophis viridiflavus) was used as our model predator. Adult green whip snakes are generalist feeders, preying upon small mammals, reptiles and birds, but neonates are highly specialized on lizards. ${ }^{139}$ Green whip snakes occur in sympatry with common lizards in their southern distribution. We used two snakes successively to prevent maintaining a single individual over a long period of time. We maintained snakes in our laboratory in a room separated from the lizards' room.

We estimated that lizards have a smaller home range than the green whip snake $\left(<1200 \mathrm{~m}^{2}\right.$ for common lizards, ${ }^{6}>10000 \mathrm{~m}^{2}$ for snakes ${ }^{139}$ ), while the mobility, estimated as sprint speed, was higher for snakes than for lizards (lizards: ${ }^{8,9} 30-90 \mathrm{~cm} \mathrm{~s}^{-1}$; snakes: ${ }^{139} 15-90 \mathrm{~cm} \mathrm{~s}^{-1}$ ). On top of dispersal, tail autotomy is another defence against predators. ${ }^{140}$

\section{Experimental setup}

We used 8 two-patch systems made of connected $1100 \mathrm{~L}$ cattle tanks similar. These systems are made of 4 cattle tanks juxtaposed and connected by a plastic pipe (diameter: $20 \mathrm{~cm}$ ). In total, systems are $7 \mathrm{~m}$ long. The first and last cattle tanks contained the same environments as home tanks and constitute origin and target patches. To go from the origin to the target patch, lizards had to cross two other tanks containing $20 \mathrm{~cm}$ of soil covered with a fake road made of tarmac roofing free of shelters. These two intermediate tanks simulated a $3.4 \mathrm{~m}$ wide road making movements between naturalized tanks harder. Preliminary trials indeed show that dispersal rate was three times lower with the fake road than with a more natural matrix and was similar to dispersal rates observed in natural populations (data not shown). In the target patch, we removed soil underneath the connecting pipe to prevent dispersing lizards moving 
back to their origin patch.

\section{Treatments}

We created 16 populations made of 7 lizards (3 females and 4 males for 10 populations and 2 females and 4 males for 6 populations). The 7 lizards were released in origin patches which represent a density within the range observed in natural populations. ${ }^{138}$ We used a 2 x 2 factorial design, crossing resource availability (RA) and predation risk (PRED) with two levels each, resulting into four replicates of each combination of treatments. We ran the four replicates in two blocks, one on July 16th and one on August 2nd. The RA and PRED treatments were applied before releasing lizards in origin patches. After a 24 hours acclimation phase with connections between patches closed, connections were opened and we monitored dispersal movements as described below.

Resource availability RA included two treatments a low and standard RA treatment. The resources were manipulated through the addition of 200 crickets in the standard RA treatment. Origin patches had some natural resources for lizards to survive in the low RAtreatment. We estimated the relative abundance of spiders and orthoptera, the main prey for common lizards. ${ }^{4}$ Our treatments created a large difference in food availability between treatments $\left(F_{1,14}=27.52, p=0.0001\right)$.

Predator cues The snake was kept in a separate room in a wood box $(50 \times 40 \times 10 \mathrm{~cm})$ featuring a clean water bowl, a hiding spot and a light bulb for basking (40 W; set on a $12 \mathrm{~L}$ : $12 \mathrm{D}$ cycle). In order to collect snake odors, we placed 40 small calcite tiles $(3 \times 3 \times 0.6 \mathrm{~cm})$ in the snake cage. The tiles were left 3 days before being transferred into the two-patch systems. Upon collection, tiles were gently rubbed against the snake belly and sides in order to saturate them with snake odour. Forty identical tiles, kept in a separate room, were used as control for the no PRED treatment. Before releasing lizards, two-patch systems with predator cues (yes PRED) received 10 tiles collected from the snake terrarium, whereas no PRED treatments received control, odour-free tiles. After each assay, all slabs were cleaned with $70 \%$ ethanol, rinsed and put back with snakes or in a control box. This procedure has been repeatedly shown to efficiently elicit antipredator responses. ${ }^{34,137,138}$

\section{Dispersal and data collection}

After the opening of corridors, we monitored dispersal daily for 9 days. To do so, we captured lizards in the target patches without disturbing the origin patches. At the end of the dispersal assay, we captured all lizards in origin and target patches. Ten lizards were found in the intermediate tanks (i.e. fake road) and, as we were unsure of their dispersal status, we excluded them from the analysis 


\section{Supplementary Figures}

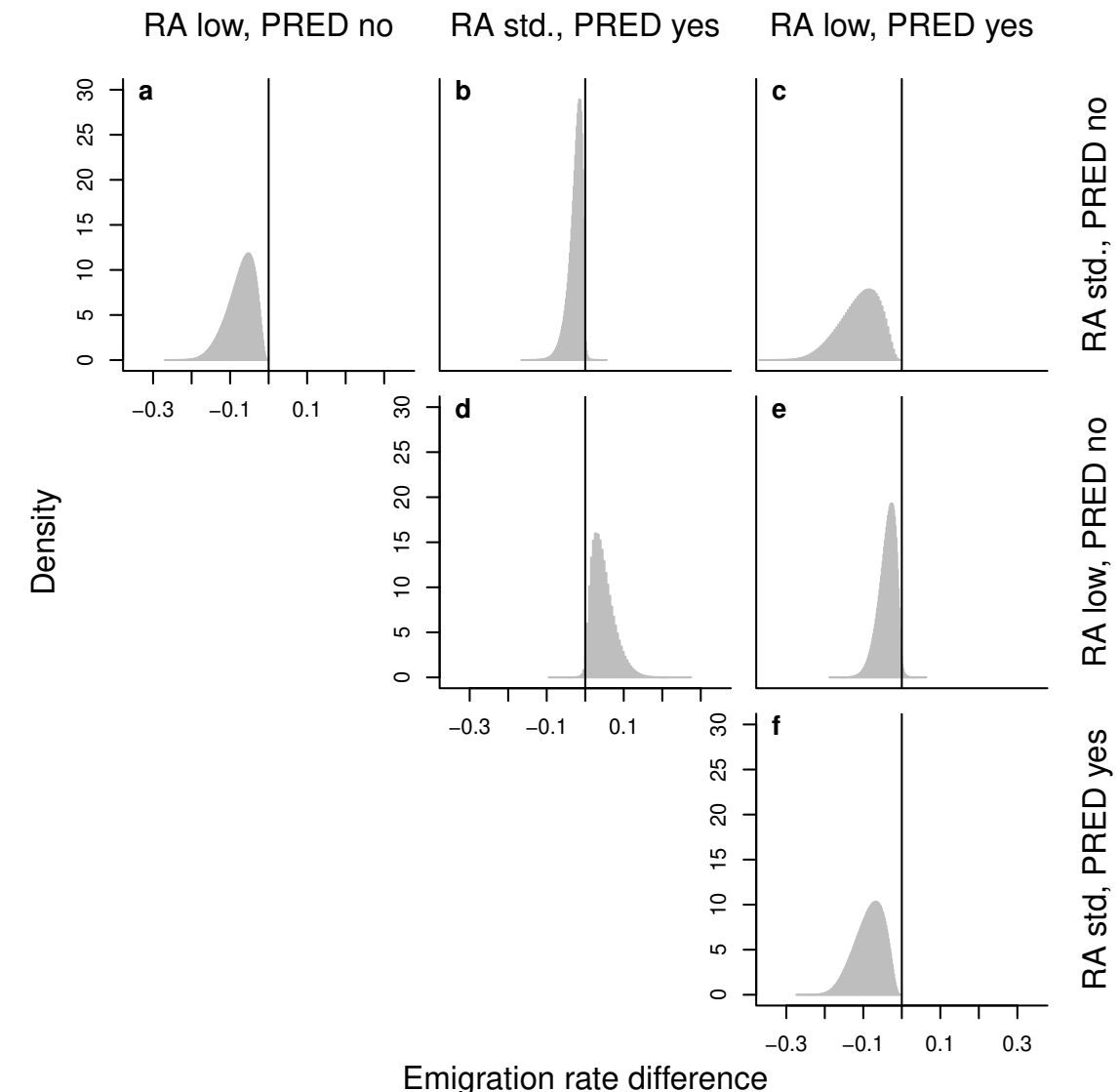

Figure S1: Pairwise differences (row header minus column header) between posterior predictive distributions of dispersal rates (back-transformed) in the four treatments for the most parsimonious, that is, additive model. 

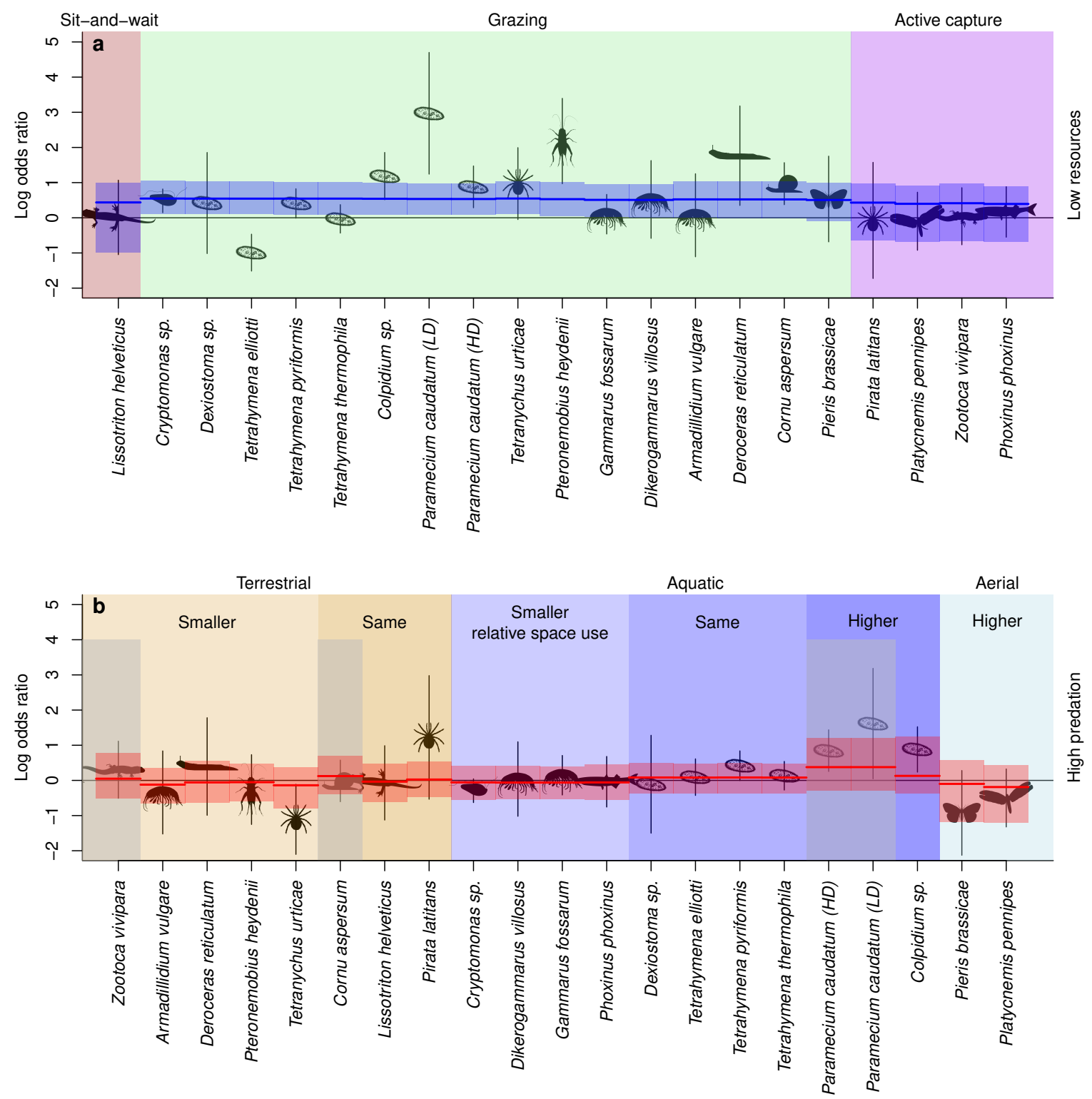

Figure S2: Effect of bottom-up resource limitation (a) and top-down predation risk (b) on emigration for each study species individually. We here show species' trends in dispersal responses (log odds ratios extracted from species specific GLMMs) with respect to species specific dispersal modulators (see Tab. S1). Note that relative space use implies focal species space use relative to the respective predator's space use. AICc-based model selection on meta-analytic mixed models confirmed the overall effect of resource limitation (Tab. S3), while the effect of predation risk was potentially modulated by prey and predator properties (Tab. S5). Grey shaded species indicate a specialized predator. We show dispersal responses (log odds ratios; logOR; black animal symbols) and confidence intervals (vertical black lines) of the resource limitation (top) and predation risk (bottom) effects per species, as well as model estimates (solid coloured lines) and confidence intervals (shaded areas) of the averaged model. 


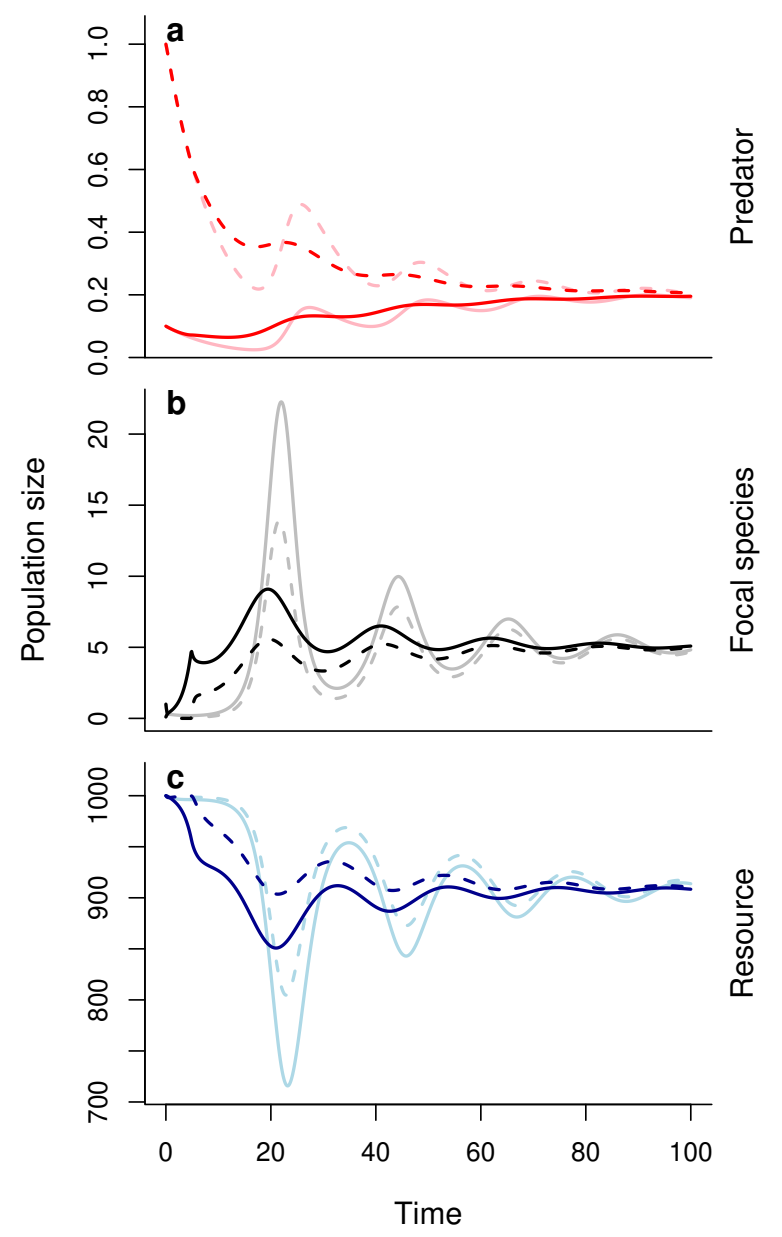

Figure S3: Consequences of context-dependent dispersal for population and metacommunity dynamics. We show the dynamics of all three tropic levels (a: top predator in red, $P$; b: focal species in black, $N$; c: resources in blue, $R$ ) in both patches (patch 1: solid lines, patch 2: dashed lines). While the RD and CDD scenarios are characterized by the same model parameters, we compare the specific scenarios in which the CDD parameters minimize the focal species population dynamics CV (as in the main text; $T_{R}=956.94$ and $T_{P}=0.12$ ) with the RD scenario that exhibits the same dispersal rate at population dynamic equilibrium (i.e, $m_{N}=1$ ). The results are not qualitatively changed, except for the covariance in predator dynamics: the $\mathrm{CV}$ of the focal species (resource, predator) population dynamics is reduced by $49 \%(48 \%, 8 \%)$ in the CDD scenario compared to RD while the covariance between dynamics in patches 1 and 2 is reduced by $88 \%$ ( $80 \%$, increased by $48 \%)$. Parameter values: $\omega=0.5, R_{0}=1000, e_{N}=0.1$, $a_{N}=0.01, d_{N}=0.1, e_{P}=0.005, a_{P}=4, d_{P}=0.1$. 


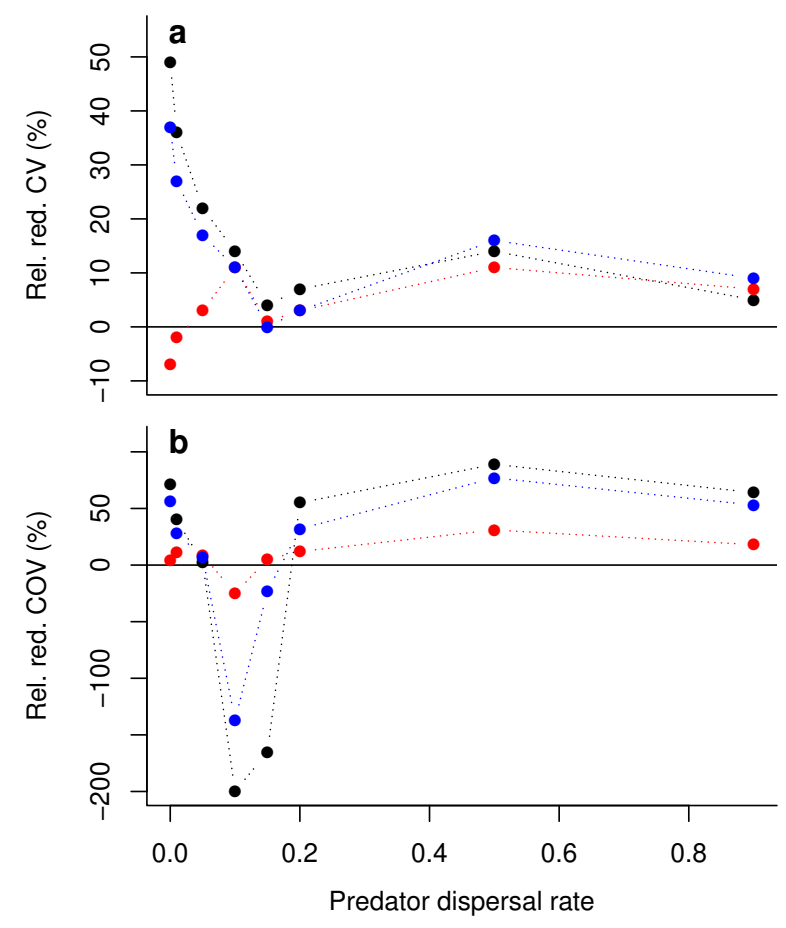

Figure S4: Consequences of context-dependent dispersal for population and metacommunity dynamics (local and regional stability) for different values of predator dispersal rates. Based on the analyses presented in Fig. 2 we show the relative reduction (Rel. red.) in coefficients of variation (a: CV) of dynamics within patches, respectively covariance (b: COV) between patches when assuming CDD with respect to resources and predators in comparison to RD. Overall, the local stabilizing effect of CDD (reduction in CV, panel a) is not qualitatively affected, the effects always remain positive, but clearly decrease as predator dispersal increases. Of course, the predator's dynamics are stabilized with increasing dispersal rates. The relationship between predator dispersal rates and the regionally stabilizing effect of CDD (reduction in COV, panel b) is slightly more complex. Fundamentally, the non-monotonic pattern occurs, because intermediate predator dispersal rates (approx. 10\% here) are not able to fully synchronize predator dynamics in the RD case between both patches, while CDD facilitates this synchronization as focal species' dynamics are synchronized. If predator dispersal is increased even further patch dynamics for predators are synchronous in the RD case and focal species CDD may desynchronize predator dynamics, at least temporarily. The desynchronization happens because, due to high initial predator densities the focal species in patch 2 goes extinct, while patch 1 it only experiences low predator and focal species densities. The focal species in patch 1 disperses less due to CDD (low predator densities) which makes the focal species increase in densities more rapidly in patch 1 while preventing rescue of patch 2 . This can be seen in the dynamics illustrated in Fig. S5. Parameter values: $\omega=0.5, R_{0}=1000, e_{N}=0.1$, $a_{N}=0.01, d_{N}=0.1, e_{P}=0.005, a_{P}=4, d_{P}=0.1$. 


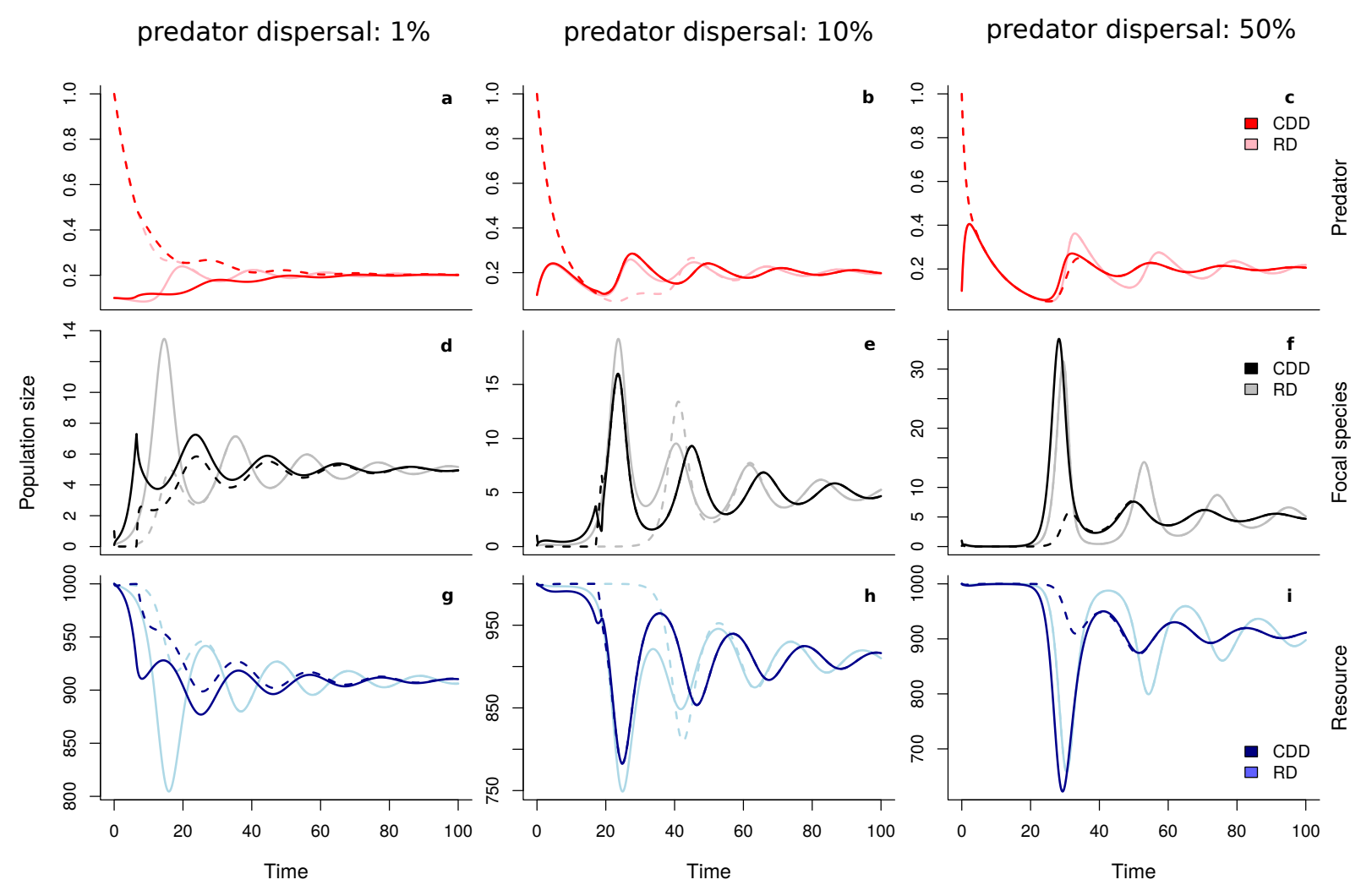

Figure S5: Example of food chain dynamics as a function of predator dispersal rates. See Fig. S4 for a systematic overview. Parameter values: $\omega=0.5, R_{0}=1000, e_{N}=0.1, a_{N}=0.01, d_{N}=0.1, e_{P}=0.005$, $a_{P}=4, d_{P}=0.1$. 
Supplementary Tables 


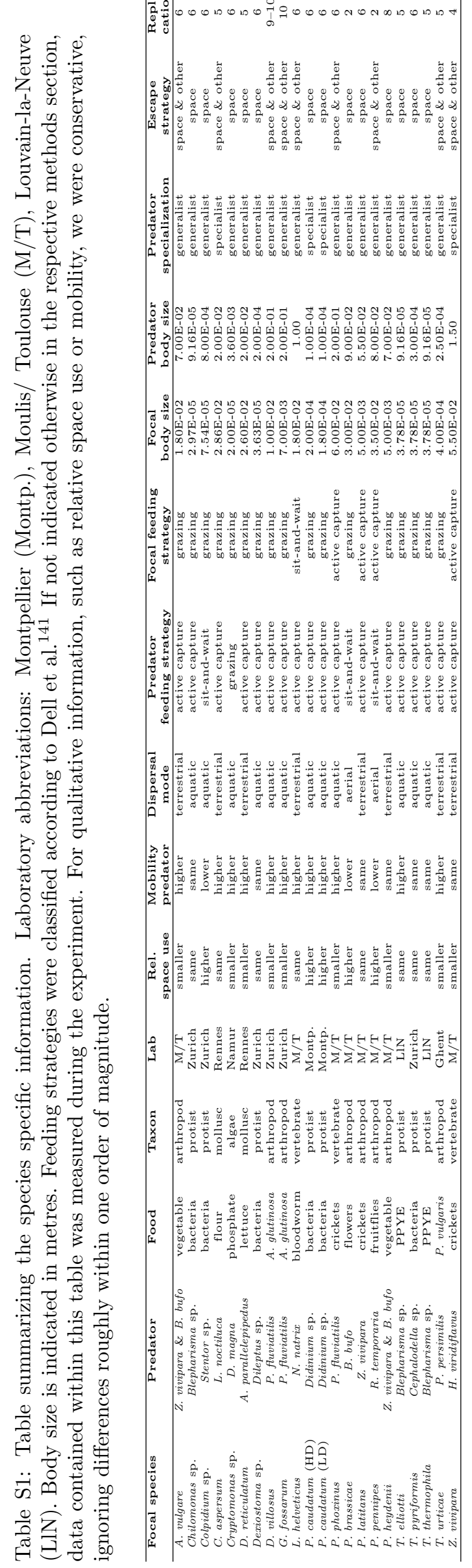


Table S2: Model selection results for the overall effect of resource limitation (RA) and predation risk (PRED). For a visualization see Fig. 1.

\begin{tabular}{lcc}
\hline Model & $\Delta$ AICc & $W_{A I C c}$ \\
\hline RA + PRED & 0 & 0.65 \\
RA * PRED & 2.07 & 0.23 \\
RA & 3.35 & 0.12 \\
PRED & 30.64 & 0 \\
1 & 33.70 & 0 \\
\hline
\end{tabular}


Table S3: Model selection results for the effect of resource limitation (RA) — additive model. We only show the top five models. For a visualization see Fig. S2.

\begin{tabular}{lcc}
\hline Model & $\Delta$ AICc & $W_{A I C c}$ \\
\hline 1 & 0 & 0.55 \\
focal feeding strategy & 2.03 & 0.20 \\
$\log ($ focal body size) & 2.53 & 0.16 \\
dispersal mode & 4.81 & 0.05 \\
$\log ($ focal body size) + focal feeding strategy & 6.03 & 0.03 \\
\hline
\end{tabular}

Table S4: Relative parameter importance (that is, sum of AICc weights of models containing the respective parameter) for the effect of resource limitation (RA) - additive model. We only show the top three parameters as the others had relative importances of zero.

\begin{tabular}{lc}
\hline Parameter & Relative importance \\
\hline focal feeding strategy & 0.23 \\
$\log ($ focal body size) & 0.19 \\
dispersal mode & 0.07 \\
\hline
\end{tabular}


Table S5: Model selection results for the effect of predation risk (PRED) — additive model. We only show the top ten models. For a visualization see Fig. S2.

\begin{tabular}{lcc}
\hline Model & $\Delta$ AICc & $W_{A I C c}$ \\
\hline 1 & 0 & 0.15 \\
dispersal mode + rel. space use & 0.15 & 0.14 \\
predator specialization & 0.36 & 0.12 \\
predator specialization + escape strategy & 1.37 & 0.07 \\
escape strategy & 2.30 & 0.05 \\
body size ratio & 2.81 & 0.04 \\
dispersal mode + rel. space use $+\log ($ focal body size) & 2.92 & 0.04 \\
$\log ($ focal body size) & 3.06 & 0.03 \\
predator specialization + body size ratio & 3.40 & 0.03 \\
$\log ($ focal body size) + predator specialization & 3.51 & 0.03 \\
\hline
\end{tabular}

Table S6: Relative parameter importance (that is, sum of AICc weights of models containing the respective parameter) for the effect of predation risk (PRED) - additive model. We only show the top nine parameters as the others had relative importances of zero.

\begin{tabular}{lc}
\hline Parameter & Relative importance \\
\hline predator specialization & 0.38 \\
dispersal mode & 0.33 \\
rel. space use & 0.26 \\
escape strategy & 0.23 \\
log(focal body size) & 0.17 \\
body size ratio & 0.15 \\
predator feeding strategy & 0.09 \\
predator mobility & 0.04 \\
relevant taxon & 0.01 \\
\hline
\end{tabular}


Table S7: Model selection results for the effect of resource limitation (RA) — interaction model. We only show the top five models.

\begin{tabular}{lcc}
\hline Model & $\Delta$ AICc & $W_{A I C c}$ \\
\hline 1 & 0 & 0.71 \\
$\log ($ focal body size) & 3.17 & 0.15 \\
dispersal mode & 4.42 & 0.08 \\
focal feeding strategy & 5.67 & 0.04 \\
dispersal mode $+\log ($ focal body size) & 8.07 & 0.01 \\
\hline
\end{tabular}

Table S8: Relative parameter importance (that is, sum of AICc weights of models containing the respective parameter) for the effect of resource limitation (RA) - interactive model. We only show the top three parameters as the others had relative importances of zero.

\begin{tabular}{lc}
\hline Parameter & Relative importance \\
\hline log(focal body size) & 0.16 \\
dispersal mode & 0.09 \\
focal feeding strategy & 0.05 \\
\hline
\end{tabular}


Table S9: Model selection results for the effect of predation risk (PRED) — interaction model. We only show the top five models.

\begin{tabular}{lcc}
\hline Model & $\Delta$ AICc & $W_{\text {AICc }}$ \\
\hline 1 & 0 & 0.31 \\
predator specialization & 0.47 & 0.25 \\
escape strategy & 2.92 & 0.07 \\
$\log ($ focal body size) & 3.09 & 0.07 \\
predator specialization $+\log$ (focal body size) & 3.20 & 0.06 \\
\hline
\end{tabular}

Table S10: Relative parameter importance (that is, sum of AICc weights of models containing the respective parameter) for the effect of predation risk (PRED) - interactive model. We only show the top 7 parameters as the others had relative importances of zero.

\begin{tabular}{lc}
\hline Parameter & Relative importance \\
\hline predator specialization & 0.43 \\
escape strategy & 0.17 \\
log(focal body size) & 0.17 \\
dispersal mode & 0.05 \\
rel. space use & 0.05 \\
predator feeding strategy & 0.03 \\
predator mobility & 0.03 \\
\hline
\end{tabular}


Table S11: Model selection results for the effect of the interaction between resource limitation and predation - interaction model. We only show the top ten models.

\begin{tabular}{lcc}
\hline Model & $\Delta$ AICc & $W_{A I C c}$ \\
\hline 1 & 0 & 0.35 \\
predator feeding strategy & 2.37 & 0.11 \\
escape strategy & 2.95 & 0.08 \\
log(focal body size) & 3.09 & 0.07 \\
predator specialization & 3.17 & 0.07 \\
rel. space use & 4.28 & 0.04 \\
predator mobility & 4.52 & 0.04 \\
focal feeding strategy & 4.62 & 0.03 \\
predator feeding strategy + escape strategy & 5.59 & 0.02 \\
dispersal mode & 5.60 & 0.02 \\
\hline
\end{tabular}

Table S12: Relative parameter importance (that is, sum of AICc weights of models containing the respective parameter) for the effect of the interaction between resource limitation and predation interactive model. We only show the top 8 parameters as the others had relative importances of zero.

\begin{tabular}{lc}
\hline Parameter & Relative importance \\
\hline predator feeding strategy & 0.20 \\
escape strategy & 0.16 \\
$\log ($ focal body size) & 0.15 \\
predator specialization & 0.14 \\
predator mobility & 0.08 \\
rel. space use & 0.06 \\
focal feeding strategy & 0.05 \\
dispersal mode & 0.04 \\
\hline
\end{tabular}


Table S13: Sensitivity analysis of the consequences of CDD in the two-patch food-chain model (Eqs. 1a-c). We here report the relative change in coefficient of variation (CV) of the temporal dynamics if dispersal is assumed to be context-dependent instead of random, that is, negative values indicate a reduction in $\mathrm{CV}$ in the CDD dynamics compared to RD. We always compare the RD-CDD scenario pair that both minimize the respective $\mathrm{CV}$ of the focal species time series. The table shows relative changes for the focal species as well as in brackets for the resource (first value) and for the predator (second value).

\begin{tabular}{ccc} 
Model parameter & $-\mathbf{5 0} \%$ of standard value & $+\mathbf{5 0} \%$ of standard value \\
\hline$\omega$ & $-43 \%(-26 \% ;+4 \%)$ & $-47 \%(-42 \% ;+1 \%)$ \\
$e_{N}$ & $-30 \%(-26 \% ;-4 \%)$ & $-37 \%(-29 \% ;-2 \%)$ \\
$a_{N}$ & $-45 \%(-29 \% ;+7 \%)$ & $-43 \%(-39 \% ;-6 \%)$ \\
$e_{P}$ & $-37 \%(-28 \% ;-5 \%)$ & $-43 \%(-36 \% ;-3 \%)$ \\
$a_{p}$ & $-39 \%(-28 \% ;-1 \%)$ \\
\hline
\end{tabular}

Table S14: Sensitivity analysis of the consequences of CDD in the two-patch food-chain model (Eqs. 1ac). We here report the relative change in covariance of the temporal dynamics in both patches if dispersal is assumed to be context-dependent instead of random, that is, negative values indicate a reduction in covariance in the CDD dynamics compared to RD. We always compare the RD-CDD scenario pair that both minimize the respective $\mathrm{CV}$ of the focal species time series. The table shows relative changes for the focal species as well as in brackets for the resource (first value) and for the predator (second value).

\begin{tabular}{ccc}
\hline Model parameter & $\mathbf{- 5 0 \%}$ of standard value & $+\mathbf{5 0} \%$ of standard value \\
\hline$\omega$ & $-73 \%(-44 \% ;-23 \%)$ & $-84 \%(-78 \% ;-6 \%)$ \\
$e_{N}$ & - & $-82 \%(-70 \% ;+7 \%)$ \\
$a_{N}$ & $+527 \%(+173 \% ;-31 \%)$ & $-94 \%(-73 \% ;+3 \%)$ \\
$e_{P}$ & $-61 \%(-42 \% ;-20 \%)$ & $-100 \%(-91 \% ;-8 \%)$ \\
$a_{p}$ & $-74 \%(-58 \% ;+26 \%)$ & $-94 \%(-81 \% ;-21 \%)$ \\
\hline
\end{tabular}




\section{Supplementary References}

[1] Legrand, D. et al. The metatron: an experimental system to study dispersal and metaecosystems for terrestrial organisms. Nat. Methods 9, 828833 (2012).

[2] Hegarty, K. G. \& Kight, S. L. Do predator cues influence turn alternation behavior in terrestrial isopods Porcellio laevis Latreille and Armadillidium vulgare Latreille? Behav. Processes 106, $168-171(2014)$

[3] Crnobrnja-Isailović, J. et al. Diet composition and food preferences in adult common toads (Bufo bufo)(Amphibia: Anura: Bufonidae). J. Herpetol. 46, 562-567 (2012).

[4] Avery, R. A. Food and feeding habits of the common lizard (Lacerta vivipara) in the west of England. J. Zool. 149, 115-121 (1966).

[5] Hoffmann, G. The influence of landmarks on the systematic search behaviour of the desert isopod Hemilepistus reaumuri. Behav. Ecol. Sociobiol. 17, 325-334 (1985).

[6] Clobert, J. et al. Determinants of dispersal behavior: the common lizard as a case study. Lizard ecology: historical and experimental perspectives 183, 183-206 (1994).

[7] Parker, A. G. \& Gittins, S. P. A note on home range in the common toad in mid-Wales and a method for tracking toads for behavioural observation. Br. J. Herpetol. 6, 7-8 (1979).

[8] Van Damme, R., Bauwens, D. \& Verheyen, R. The thermal dependence of feeding behaviour, food consumption and gut-passage time in the lizard Lacerta vivipara Jacquin. Funct. Ecol. 5, 507-517 (1991).

[9] Sorci, G., Swallow, J. G., Garland Jr, T. \& Clobert, J. Quantitative genetics of locomotor speed and endurance in the lizard Lacerta vivipara. Physiol. Zool. 68, 698-720 (1995).

[10] Beck, C. W. \& Congdon, J. D. Effects of age and size at metamorphosis on performance and metabolic rates of southern toad, Bufo terrestris, metamorphs. Funct. Ecol. 14, 32-38 (2000).

[11] Dailey, T. M., Claussen, D. L., Ladd, G. B. \& Buckner, S. T. The effects of temperature, desiccation, and body mass on the locomotion of the terrestrial isopod, Porcellio laevis. Comp. Biochem. Physiol. A-Mol. Integr. Physiol. 153, 162-166 (2009).

[12] Broly, P., Deneubourg, J.-L. \& Devigne, C. Benefits of aggregation in woodlice: a factor in the terrestrialization process? Insect. Soc. 60, 419-435 (2013).

[13] Iorgu, I. Ş. \& Iorgu, E. I. Bush-crickets, crickets and grasshoppers from Moldavia (Romania) (Pim, 2008). 
[14] Hao-Yu, L., Li-Mei, L. \& Fu-Ming, S. Checklist of nemobiinae from china (orthoptera: Trigonidiidae). Int. J. Fauna Biol. Stud. 3, 103-108 (2016).

[15] Linzey, D. W., Deel, A. C., Adams, S. J. \& Linzey, J. B. Spring and summer foods of Bufo marinus (amphibia: Bufonidae) and Eleutherodactylus johnstonei (amphibia: Leptodactylidae) in bermuda. J. Elisha Mitchell Sci. Soc. 114, 125-136 (1998).

[16] Brouwers, N. \& Newton, A. Movement analyses of wood cricket (Nemobius sylvestris)(orthoptera: Gryllidae). Bull. Entomol. Res. 100, 623-634 (2010).

[17] Dangles, O., Ory, N., Steinmann, T., Christides, J.-P. \& Casas, J. Spider's attack versus cricket's escape: velocity modes determine success. Anim. Behav. 72, 603-610 (2006).

[18] Framenau, V. W. Gender specific differences in activity and home range reflect morphological dimorphism in wolf spiders (Araneae, Lycosidae). J. Arachnol. 33, 334-346 (2005).

[19] Apontes, P. \& Brown, C. A. Between-sex variation in running speed and a potential cost of leg autotomy in the wolf spider Pirata sedentarius. Am. Midl. Nat. 154, 115-125 (2005).

[20] Griffiths, R. A. Seasonal behaviour and intrahabitat movements in an urban population of Smooth newts, Triturus vulgaris (Amphibia: Salamandridae). J. Zool. 203, 241-251 (1984).

[21] Hailey, A. \& Davies, P. M. C. Lifestyle, latitude and activity metabolism of natricine snakes. J. Zool. 209, 461-476 (1986).

[22] Gregory, P. T. \& Isaac, L. A. Food habits of the grass snake in southeastern England: Is Natrix natrix a generalist predator? J. Herpetol. 38, 88-95 (2004).

[23] Consul, A., Eger, S. \& Kwet, A. The grass snake, Natrix natrix natrix (Squamata: Colubridae), as a predator of the Great Ramshorn Snail, Planorbarius c. corneus (Gastropoda: Planorbidae). Salamandra 45, 50-52 (2009).

[24] Verell, P. A. The directionality of migrations of amphibians to and from a pond in southern England, with particular reference to the smooth newt, Triturus vulgaris. Amphibia-Reptilia 8 , 93-100 (1987).

[25] Hehl-Lange, S. Structural elements of the visual landscape and their ecological functions. Landscape Urban Plann. 54, 107-115 (2001).

[26] Madsen, T. Movements, home range size and habitat use of radio-tracked grass snakes (Natrix natrix) in southern Sweden. Copeia 3, 707-713 (1984).

[27] Reading, C. J. \& Jofré, G. M. Habitat selection and range size of grass snakes Natrix natrix in an agricultural landscape in southern England. Amphibia-Reptilia 30, 379-388 (2009). 
[28] Wilson, R. S. Consequences of metamorphosis for the locomotor performance and thermal physiology of the newt Triturus cristatus. Physiol. Biochem. Zool. 78, 967-975 (2005).

[29] Gvoždík, L. \& Van Damme, R. Triturus newts defy the running-swimming dilemma. Evolution 60, 2110-2121 (2006).

[30] Isaac, L. A. \& Gregory, P. T. Aquatic versus terrestrial locomotion: comparative performance of two ecologically contrasting species of European natricine snakes. J. Zool. 273, 56-62 (2007).

[31] Brodie, E. D. Salamander antipredator postures. Copeia 3, 523-535 (1977).

[32] Yotsu-Yamashita, M., Mebs, D., Kwet, A. \& Schneider, M. Tetrodotoxin and its analogue 6epitetrodotoxin in newts (Triturus spp.; Urodela, Salamandridae) from southern Germany. Toxicon 50, 306-309 (2007).

[33] Cote, J. \& Clobert, J. Social information and emigration: lessons from immigrants. Ecol. Lett. 10, 411-417 (2007).

[34] Teyssier, A., Bestion, E., Richard, M. \& Cote, J. Partners personality types and mate preferences: predation risk matters. Behav. Ecol. 25, 723-733 (2014).

[35] Madison, D. M., Maerz, J. C. \& McDarby, J. H. Optimization of predator avoidance by salamanders using chemical cues: diet and diel effects. Ethology 105, 1073-1086 (1999).

[36] Maerz, J. C., Panebianco, N. L. \& Madison, D. M. Effects of predator chemical cues and behavioral biorhythms on foraging, activity of terrestrial salamanders. J. Chem. Ecol. 27, 1333-1344 (2001).

[37] Sullivan, A. M., Maerz, J. C. \& Madison, D. M. Anti-predator response of red-backed salamanders (Plethodon cinereus) to chemical cues from garter snakes (Thamnophis sirtalis): laboratory and field experiments. Behav. Ecol. Sociobiol. 51, 227-233 (2002).

[38] Winandy, L. \& Denoël, M. Cues from introduced fish alter shelter use and feeding behaviour in adult alpine newts. Ethology 119, 121-129 (2013).

[39] Joly, P. \& Miaud, C. How does a newt find its pond? the role of chemical cues in migrating newts (Triturus alpestris). Ethol. Ecol. Evol. 5, 447-447 (1993).

[40] Altermatt, F. et al. Big answers from small worlds: a user's guide for protist microcosms as a model system in ecology and evolution. Methods Ecol. Evol. 6, 218-231 (2015).

[41] Fronhofer, E. A., Klecka, J., Melián, C. \& Altermatt, F. Condition-dependent movement and dispersal in experimental metacommunities. Ecol. Lett. 18, 954-963 (2015). 
[42] Fronhofer, E. A., Gut, S. \& Altermatt, F. Evolution of density-dependent movement during experimental range expansions. J. Evol. Biol. 30, 2165-2176 (2017).

[43] Pennekamp, F., Schtickzelle, N. \& Petchey, O. L. Bemovi, software for extracting behavior and morphology from videos, illustrated with analyses of microbes. Ecol. Evol. 5, 2584-2595 (2015).

[44] Welter-Schultes, F. European Non-Marine Molluscs, a Guide for Species Identification (Planet Poster Editions, Göttingen, Germany, 2012).

[45] Dahirel, M., Vardakis, M., Ansart, A. \& Madec, L. Density-dependence across dispersal stages in a hermaphrodite land snail: insights from discrete choice models. Oecologia 181, 1117-1128 (2016).

[46] Dan, N. Studies on the growth and ecology of Helix aspersa Müller. Ph.D. thesis, University of Manchester, Manchester, UK (1978).

[47] Symondson, W. O. C. Coleoptera (Carabidae, Staphylinidae, Lampyridae, Drilidae and Silphidae) as pedators of terrestrial gastropods. In Barker, G. (ed.) Natural enemies of terrestrial molluscs, 37-84 (CABI, Wallingford, UK., 2004).

[48] De Cock, R. Biology and behaviour of European lampyrids. In Meyer-Rochow, V. B. (ed.) Bioluminescence in Focus: a Collection of Illuminating Essays, 163-200 (Research Signpost, Kerala, India, 2009).

[49] Dahirel, M. et al. Movement propensity and ability correlate with ecological specialization in european land snails: comparative analysis of a dispersal syndrome. J. Anim. Ecol. 84, 228-238 (2015).

[50] Chase, R. Sensory organs and the nervous system. In Barker, G. (ed.) The biology of terrestrial molluscs, 179-211 (CABI, Wallingford, UK., 2001).

[51] Bursztyka, P. et al. The foraging behaviour of the slug Deroceras reticulatum (Müller, 1774) is modified in the presence of cuticular scents from a carabid beetle. J. Molluscan Stud. 82, 314-319 (2016).

[52] Kilham, S. S., Kreeger, D. A., Lynn, S. G., Goulden, C. E. \& Herrera, L. COMBO: a defined freshwater culture medium for algae and zooplankton. Hydrobiologia 377, 147-159 (1998).

[53] Arvola, L., Salonen, K., Kankaala, P. \& Lehtovaara, A. Vertical distributions of bacteria and algae in a steeply stratified humic lake under high grazing pressure from Daphnia longispina. Hydrobiologia 229, 253-269 (1992).

[54] Latta, I. V., Leigh, C., O’Donnell, R. P. \& Pfrender, M. E. Vertical distribution of Chlamydomonas changes in response to grazer and predator kairomones. Oikos 118, 853-858 (2009). 
[55] Harvey, E. L. \& Menden-Deuer, S. Predator-induced fleeing behaviors in phytoplankton: a new mechanism for harmful algal bloom formation? PLoS One 7, e46438 (2012).

[56] Barker, G. M. (ed.) Molluscs as Crop Pests (CABI, Wallingford, UK, 2002).

[57] Kappes, H. et al. Response of snails and slugs to fragmentation of lowland forests in NW germany. Landsc. Ecol. 24, 685-697 (2009).

[58] Armsworth, C. G., Bohan, D. A., Powers, S. J., Glen, D. M. \& Symondson, W. O. C. Behavioural responses by slugs to chemicalsfrom a generalist predator. Anim. Behav. 69, 805-811 (2005).

[59] Duflot, R., Ernoult, A., Burel, F. \& Aviron, S. Landscape level processes driving carabid crop assemblage in dynamic farmlands. Popul. Ecol. 58, 265-275 (2016).

[60] Eyre, M. D., McMillan, S. D. \& Critchley, C. N. R. Ground beetles (Coleoptera, Carabidae) as indicators of change and pattern in the agroecosystem: Longer surveys improve understanding. Ecol. Indic. 68, 82-88 (2016).

[61] Symondson, W. O. C. The effects of crop development upon slug distribution and control by Abax parallelepipedus (Coleoptera: Carabidae). Ann. Appl. Biol. 123, 449-457 (1993).

[62] Symondson, W. O. C. \& Liddell, J. E. The detection of predation by Abax parallelepipedus and Pterostichus madidus (Coleoptera: Carabidae) on Mollusca using a quantitative ELISA. Bull. Entomol. Res. 83, 641-647 (1993).

[63] South, A. Biology and ecology of Agriolimax reticulatus (Müll.) and other slugs: spatial distribution. J. Anim. Ecol. 403-417 (1965).

[64] Charrier, S., Petit, S. \& Burel, F. Movements of Abax parallelepipedus (Coleoptera, Carabidae) in woody habitats of a hedgerow network landscape: a radio-tracing study. Agric. Ecosyst. Environ. 61, 133-144 (1997).

[65] Forsythe, T. G. Running and pushing in relationship to hind leg structure in some carabidae (coleoptera). Coleopt. Bull. 353-378 (1981).

[66] Bursztyka, P., Saffray, D., Lafont-Lecuelle, C., Brin, A. \& Pageat, P. Chemical compounds related to the predation risk posed by malacophagous ground beetles alter self-maintenance behavior of naive slugs (Deroceras reticulatum). PLoS One 8, e79361 (2013).

[67] Symondson, W. O. C., Glen, D. M., Wiltshire, C. W., Langdon, C. J. \& Liddell, J. E. Effects of cultivation techniques and methods of straw disposal on predation by Pterostichus melanarius (Coleoptera: Carabidae) upon slugs (Gastropoda: Pulmonata) in an arable field. J. Appl. Ecol. 741-753 (1996). 
[68] Macneil, C., Dick, J. T. A. \& Elwood, R. W. The dynamics of predation on Gammarus spp.(Crustacea: Amphipoda). Biol. Rev. 74, 375-395 (1999).

[69] Diehl, S. Effects of habitat structure on resource availability, diet and growth of benthivorous perch, Perca fluviatilis. Oikos 67, 403-414 (1993).

[70] Hughes, D. A. Some factors affecting drift and upstream movements of Gammarus pulex. Ecology 51, 301-305 (1970).

[71] Elliott, J. A comparative study of the dispersal of 10 species of stream invertebrates. Freshwater Biol. 48, 1652-1668 (2003).

[72] van den Brink, F. W. B., van der Velde, G. \& Bij De Vaate, A. Amphipod invasion on the Rhine. Nature 352, 576-576 (1991).

[73] Allen, K. R. The food and migration of the perch (Perca fluviatilis) in Windermere. J. Anim. Ecol. 4, 264-273 (1935).

[74] Persson, L. The effects of temperature and meal size on the rate of gastric evacuation in perch (Perca fluviatilis) fed on fish larvae. Freshwater Biol. 11, 131-138 (1981).

[75] Jamet, J.-L. \& Desmolles, F. Growth, reproduction and condition of roach (Rutilus rutilus (1.)), perch (Perca fluviatilis, l.) and ruffe (Gymnocephalus cernuus (1.)) in eutrophic Lake Aydat (France). Int. Rev. Hydrobiol. 79, 305-322 (1994).

[76] Kelleher, B. et al. Effects of exotic amphipod invasions on fish diet in the Lower Rhine. Archiv für Hydrobiologie 143, 363-382 (1998).

[77] Nyman, A.-M., Hintermeister, A., Schirmer, K. \& Ashauer, R. The insecticide imidacloprid causes mortality of the freshwater amphipod Gammarus pulex by interfering with feeding behavior. PLoS One 8, e62472 (2013).

[78] Haeckel, J.-W., Meijering, M. P. D. \& Rusetzki, H. Gammarus fossarum Koch als Fallaubzersetzer in Waldbachen. Freshwater Biol. 3, 241-249 (1973).

[79] Dunn, A. M., Dick, J. T. A. \& Hatcher, M. J. The less amorous Gammarus: predation risk affects mating decisions in Gammarus duebeni (amphipoda). Anim. Behav. 76, 1289-1295 (2008).

[80] Schäffer, M., Winkelmann, C., Hellmann, C. \& Benndorf, J. Reduced drift activity of two benthic invertebrate species is mediated by infochemicals of benthic fish. Aquat. Ecol. 47, 99-107 (2013).

[81] Szokoli, F., Winkelmann, C., Berendonk, T. U. \& Worischka, S. The effects of fish kairomones and food availability on the predator avoidance behaviour of Gammarus pulex. Fundam. Appl. Limnol. 186, 249-258 (2015). 
[82] Veilleux, B. An analysis of the predatory interaction between Paramecium and Didinium. J. Anim. Ecol. 48, 787-803 (1979).

[83] Hammill, E., Fitzjohn, R. G. \& Srivastava, D. S. Conspecific density modulates the effect of predation on dispersal rates. Oecologia 178, 11491158 (2015).

[84] Kuhlmann, H.-W. Escape response of Euplotes octocarinatus to turbellarian predators. Archiv für Protistenkunde 144, 163-171 (1994).

[85] Kusch, J. Behavioural and morphological changes in ciliates induced by the predator Amoeba proteus. Oecologia 96, 354-359 (1993).

[86] Nidelet, T. \& Kaltz, O. Direct and correlated responses to selection in a host-parasite system: testing for the emergence of genotype specificity. Evolution 61, 1803-1811 (2007).

[87] Fronhofer, E. A. \& Altermatt, F. Eco-evolutionary feedbacks during experimental range expansions. Nat. Commun. 6, 6844 (2015).

[88] Fellous, S., Quillery, E., Duncan, A. B. \& Kaltz, O. Parasitic infection reduces dispersal of ciliate host. Biol. Lett. 7, 327-329 (2010).

[89] Radinger, J. \& Wolter, C. Patterns and predictors of fish dispersal in rivers. Fish. Fish. 15, 456-473 (2014).

[90] Mee, J. A., Brauner, C. J. \& Taylor, E. B. Repeat swimming performance and its implications for inferring the relative fitness of asexual hybrid dace (Pisces: Phoxinus) and their sexually reproducing parental species. Physiol. Biochem. Zool. 84, 306-315 (2011).

[91] Mallen-Cooper, M. Swimming ability of adult golden perch, Macquaria ambigua (Percichthyidae), and adult silver perch, Bidyanus bidyanus (Teraponidae), in an experimental vertical-slot fishway. Mar. Freshwater Res. 45, 191-198 (1994).

[92] Magurran, A. E. \& Girling, S. L. Predator model recognition and response habituation in shoaling minnows. Anim. Behav. 34, 510-518 (1986).

[93] Murphy, K. E. \& Pitcher, T. J. Predator attack motivation influences the inspection behaviour of European minnows. J. Fish Biol. 50, 407-417 (1997).

[94] Magurran, A. E. Acquired recognition of predator odour in the European minnow (Phoxinus phoxinus). Ethology 82, 216-223 (1989).

[95] Feltwell, J. Large White Butterfly: The Biology, Biochemistry, and Physiology of Pieris Brassicae (Dr. W. Junk Publishers, The Hague, Netherlands, 1982). 
[96] Jones, R. E., Gilbert, N., Guppy, M. \& Nealis, V. Long-distance movement of Pieris rapae. J. Anim. Ecol. 49, 629-642 (1980).

[97] Shreeve, T. G. Flight patterns of butterfly species in woodlands. Oecologia 51, 289-293 (1981).

[98] Srygley, R. B. \& Dudley, R. Correlations of the position of center of body mass with butterfly escape tactics. J. Exp. Biol. 174, 155-166 (1993).

[99] Dijkstra, K.-D. B. \& Lewington, R. Field Guide to the Dragonflies of Britain and Europe (British Wildlife Publishing Ltd, Dorset, UK, 2006).

[100] Corbet, P. S. Dragonflies: Behavior and Ecology of Odonata (Cornell University Press, Ithaca, United States, 1999).

[101] Bennett, S. \& Mill, P. J. Pre-and post-maturation survival in adults of the damselfly Pyrrhosoma nymphula (Zygoptera: Coenagrionidae). J. Zool. 235, 559-575 (1995).

[102] Loman, J. Site tenacity, within and between summers, of Rana arvalis and Rana temporaria. Alytes 12, 15-29 (1994).

[103] Haapanen, A. Site tenacity of the common frog (Rana temporaria 1.) and the moor frog ( $R$. arvalis nilss.). Ann. Zool. Fennici 7, 61-66 (1970).

[104] Miller, K., Monteforte, P. B. \& Landis, L. F. Scaling of locomotor performance and enzyme activity in the leopard frog, Rana pipiens. Herpetologica 49, 383-392 (1993).

[105] Rüppell, G. Kinematic analysis of symmetrical flight manoeuvres of Odonata. J. Exp. Biol. 144, $13-42$ (1989).

[106] Rehfeldt, G. E. Anti-predator strategies in oviposition site selection of Pyrrhosoma nymphula (Zygoptera: Odonata). Oecologia 85, 233-237 (1990).

[107] Martens, A. Group oviposition in three platycnemidid species (Odonata: Platycnemididae). Int. J. Odonatol. 5, 75-80 (2002).

[108] Legrand, D. et al. Ranking the ecological causes of dispersal in a butterfly. Ecography 38, 822-831 (2015).

[109] Trochet, A. et al. Population sex ratio and dispersal in experimental, two-patch metapopulations of butterflies. J. Anim. Ecol. 82, 946-955 (2013).

[110] Shapiro, A. M. The role of sexual behavior in density-related dispersal of pierid butterflies. Am. Nat. 104, 367-372 (1970). 
[111] Hardersen, S. Dragonfly (odonata) communities at three lotic sites with different hydrological characteristics. Ital. J. Zoolog. 75, 271-283 (2008).

[112] Sukhacheva, G. Study of the natural diet of adult dragonflies using an immunological method. Odonatologica 25, 397-403 (1996).

[113] Debecker, S., Sanmartín-Villar, I., Guinea-Luengo, M., Cordero-Rivera, A. \& Stoks, R. Integrating the pace-of-life syndrome across species, sexes and individuals: covariation of life history and personality under pesticide exposure. J. Anim. Ecol. 85, 726-738 (2016).

[114] Blaustein, L. Oviposition site selection in response to risk of predation: evidence from aquatic habitats and consequences for population dynamics and community structure. In Evolutionary Theory and Processes: Modern Perspectives, 441-456 (Springer, 1999).

[115] Pennekamp, F., Mitchell, K. A., Chaine, A. S. \& Schtickzelle, N. Dispersal propensity in Tetrahymena thermophila ciliates — a reaction norm perspective. Evolution 68, 2319-2330 (2014).

[116] Fjerdingstad, E., Schtickzelle, N., Manhes, P., Gutierrez, A. \& Clobert, J. Evolution of dispersal and life history strategies — Tetrahymena ciliates. BMC Evol. Biol. 7, 133 (2007).

[117] Schtickzelle, N., Fjerdingstad, E. J., Chaine, A. \& Clobert, J. Cooperative social clusters are not destroyed by dispersal in a ciliate. BMC Evol. Biol. 9, 251 (2009).

[118] Chaine, A. S., Schtickzelle, N., Polard, T., Huet, M. \& Clobert, J. Kin-based recognition and social aggregation in a ciliate. Evolution 64, 1290-1300 (2010).

[119] Jacob, S. et al. Cooperation-mediated plasticity in dispersal and colonization. Evolution 70, 2336$2345(2016)$.

[120] Jacob, S., Chaine, A. S., Schtickzelle, N., Huet, M. \& Clobert, J. Social information from immigrants: multiple immigrant based sources of information for dispersal decisions in a ciliate. $J$. Anim. Ecol. 85, 1373-1383 (2015).

[121] Jacob, S. et al. Gene flow favours local adaptation under habitat choice in ciliate microcosms. Nat. Ecol. Evol. 1, 1407 (2017).

[122] Jacob, S., Clobert, J., Legrand, D., Schtickzelle, N. \& Chaine, A. S. Social information in cooperation and dispersal in Tetrahymena. In Witzany, G. \& Nowacki, M. (eds.) Biocommunication of Ciliates, 235-252 (Springer, 2016).

[123] Pennekamp, F. \& Schtickzelle, N. Implementing image analysis in laboratory-based experimental systems for ecology and evolution: a hands-on guide. Methods Ecol. Evol. 4, 483-492 (2013). 
[124] Van Leeuwen, T. et al. Mitochondrial heteroplasmy and the evolution of insecticide resistance: non-mendelian inheritance in action. Proc. Natl. Acad. Sci. U. S. A. 105, 5980-5985 (2008).

[125] Fasulo, T. R. \& Denmark, H. A. Twospotted Spider Mite, Tetranychus urticae Koch (Arachnida: Acari: Tetranychidae) (University of Florida Cooperative Extension Service, Institute of Food and Agricultural Sciences, EDIS, 2000).

[126] Sabelis, M. W. \& Van der Meer, J. Local dynamics of the interaction between predatory mites and two-spotted spider mites. In Merz, J. A. J. \& Diekman, O. (eds.) Dynamics of physiologically structured populations, vol. 68 of Springer lecture notes in biomathematics, 322-343 (Springer, New York, 1986).

[127] Grostal, P. \& Dicke, M. Direct and indirect cues of predation risk influence behavior and reproduction of prey: a case for acarine interactions. Behav. Ecol. 10, 422-427 (1999).

[128] Dicke, M. \& Sabelis, M. W. How plants obtain predatory mites as bodyguards. Neth. J. Zool. 38, 148-165 (1987).

[129] Pallini, A., Janssen, A. \& Sabelis, M. Spider mites avoid plants with predators. Exp. Appl. Acarol. 23, 803-815 (1999).

[130] Lemos, F. et al. Spider mite web mediates anti-predator behaviour. Exp. Appl. Acarol. 52, 1-10 (2010).

[131] Kennedy, G. G. \& Smitley, D. R. Dispersal. In Helle, W. \& Sabelis, M. (eds.) Spider mites: their biology, natural enemies and control, 233-251 (Elsevier, Amsterdam, 1985).

[132] Li, J. B. \& Margolies, D. C. Effects of mite age, mite density, and host quality on aerial dispersal behavior in the 2-spotted spider-mite. Entomol. Exp. Appl. 68, 79-86 (1993).

[133] Bitume, E. V. et al. Density and genetic relatedness increase dispersal distance in a subsocial organism. Ecol. Lett. 16, 430-437 (2013).

[134] Kant, M. R., Ament, K., Sabelis, M. W., Haring, M. A. \& Schuurink, R. C. Differential timing of spider mite-induced direct and indirect defenses in tomato plants. Plant Physiol. 135, 483-495 (2004).

[135] Kriesch, S. \& Dicke, M. Avoidance of predatory mites by the two-spotted spider mite Tetranychus urticae: the role of infochemicals. In Proceedings of the Section Experimental and Applied Entomology - Netherlands Entomological Society, vol. 8, 121-126 (Netherlands Entomological Society, 1997). 
[136] Dicke, M. \& Grostal, P. Chemical detection of natural enemies by arthropods: an ecological perspective. Annu. Rev. Ecol. Syst. 32, 1-23 (2001).

[137] Bestion, E., Teyssier, A., Aubret, F., Clobert, J. \& Cote, J. Maternal exposure to predator scents: offspring phenotypic adjustment and dispersal. Proc. R. Soc. B-Biol. Sci. 281, 20140701 (2014).

[138] Bestion, E., Clobert, J. \& Cote, J. Dispersal response to climate change: scaling down to intraspecific variation. Ecol. Lett. 18, 1226-1233 (2015).

[139] Lelièvre, H., Le Hénanff, M., Blouin-Demers, G., Naulleau, G. \& Lourdais, O. Thermal strategies and energetics in two sympatric colubrid snakes with contrasted exposure. J. Comp. Physiol. B 180, 415-425 (2010).

[140] Bateman, P. \& Fleming, P. To cut a long tail short: a review of lizard caudal autotomy studies carried out over the last 20 years. J. Zool. 277, 1-14 (2009).

[141] Dell, A. I., Pawar, S. \& Savage, V. M. Temperature dependence of trophic interactions are driven by asymmetry of species responses and foraging strategy. J. Anim. Ecol. 83, 70-84 (2014). 